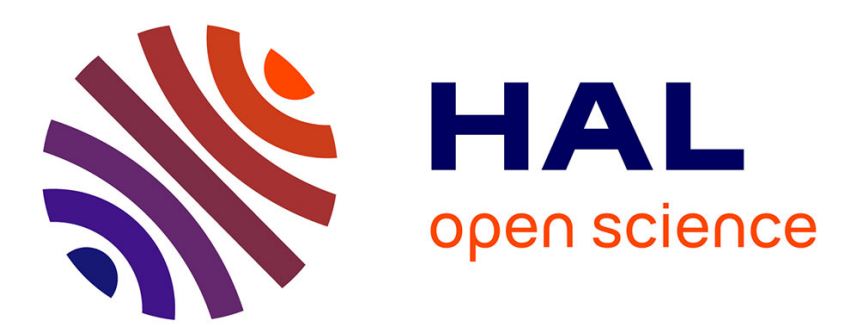

\title{
Prediction of the vibro-acoustic behavior of a submerged shell non periodically stiffened by internal frames
}

Laurent Maxit, Jean-Marc Ginoux

\section{To cite this version:}

Laurent Maxit, Jean-Marc Ginoux. Prediction of the vibro-acoustic behavior of a submerged shell non periodically stiffened by internal frames. Journal of the Acoustical Society of America, 2010, 128 (1), pp.137-151. 10.1121/1.3436526 . hal-02414231

\section{HAL Id: hal-02414231 \\ https://hal.science/hal-02414231}

Submitted on 16 Dec 2019

HAL is a multi-disciplinary open access archive for the deposit and dissemination of scientific research documents, whether they are published or not. The documents may come from teaching and research institutions in France or abroad, or from public or private research centers.
L'archive ouverte pluridisciplinaire HAL, est destinée au dépôt et à la diffusion de documents scientifiques de niveau recherche, publiés ou non, émanant des établissements d'enseignement et de recherche français ou étrangers, des laboratoires publics ou privés. 


\title{
Prediction of the vibro-acoustic behavior of a submerged shell non periodically stiffened by internal frames.
}

\author{
$\underline{\text { Laurent MAXIT (Corresponding author) }}$ \\ Laboratoire Vibrations - Acoustique - INSA Lyon \\ 25 bis, avenue Jean Capelle \\ 69621 Villeurbanne Cedex, FRANCE \\ Email: laurent.maxit@insa-lyon.fr \\ Phone: (+33) 472438082 \\ Fax: (+33) 472438712 \\ Jean-Marc GINOUX \\ DGA (Defence Agency) \\ CTSN/SDP/ASN-PFN/FUR \\ Avenue de la Tour Royale \\ BP 40915, 83050 Toulon Cedex, FRANCE \\ jean-marc.ginoux @dga.defense.gouv.fr \\ Phone: (+33) 494162207 \\ Fax: (+33) 494162025
}

PACS: 43.40.Ey, 43.40.Rj, 43.30.Jx

Running title: Radiation from a stiffened shell 


\section{Abstract:}

This paper describes the development of a numerical model to predict the vibro-acoustic behaviour of an externally fluid loaded shell with non-uniformly space stiffeners and transversal bulkheads. This model constitutes an extension of the existing semi-analytic capability in predicting the acoustics of axisymmetric structures. It is based on the Circumferential Admittance Approach (CAA) which consists in substructuring the problem so that the fluid loaded shell constitutes one subsystem and the frames constitute other independent subsystems. These subsystems are coupled together by assembling the circumferential admittances that characterise each uncoupled subsystem. Different numerical approaches can be used to estimate these admittances. The Standard Finite Element code is well adapted for evaluating the admittances of the internal frames whatever their cross-section geometries and material properties. Classical discretization methods such as Finite Elements and Boundary Elements are too time-consuming for the fluid loaded shell. To avoid this obstacle, three different approaches with different degrees of approximation are proposed to estimate the shell admittances. Comparisons with a reference case are proposed to evaluate the accuracy and the efficiency of each of these three approaches. With the optimal approach, CAA gives very good results in satisfactory computing time. It is well-adapted for analysing the behaviour of a submarine pressure hull in a wide frequency range of interest. 


\section{INTRODUCTION}

The acoustic performances of submarine hulls affect different operational capacities of submarines [1]:

- the radiated noise in the far field influences the acoustic stealth of the submarine;

- the radiated noise in the near field (self-noise) can reduce Sonar performances;

- the acoustic wave reflection on the hull plays a role in the active Sonar threat (target strength). Modelling the vibro-acoustic behaviour of the submarine hull can help us to understand the physical phenomena involved in its acoustic performance, in order to quantify and to improve it. To achieve good representativeness of a submarine pressure hull, the model should at least take into account: (a) the cylindrical hull and its interaction with the external fluid (water); (b) the bulkheads between the different compartments and the ends of the pressure hull; and (c) the stiffeners whose spacing can vary along the shell. The frequency range of interest for submarine applications is wide, from one $\mathrm{Hz}$ to several $\mathrm{KHz}$. The numerical model should be able to give results in this frequency range for a shell with the size of a submarine hull, which is the main challenge addressed in this paper. Indeed, numerical methods such as the Finite Element and Boundary Element methods generally used to analysis complex systems, are limited to low frequencies (below $100 \mathrm{~Hz}$ ) by the number of degrees of freedom. This increases dramatically as the frequency increases (see [2-5]). The development of a dedicated model is an alternative for overcoming this obstacle. We propose in this paper to develop a model of a shell immerged in a fluid and stiffened by the ring stiffeners and the axisymmetric bulkheads of a submarine pressure hull. The circumferential admittance approach will be used to assemble a numerical model of a fluid loaded shell with the finite element models of its internal frames. 
Up to now, numerous scientists have already developed models to analyse the vibro-acoustic behaviour of fluid loaded stiffened shells. Former works generally considered a plate instead of a shell, permitting the analysis of certain phenomenological links with the periodicity of the structure (e.g. stop band/pass band effect) [6,7] and the effect of stiffeners on acoustic radiation [8]. Concerning shell models, Langley [9] developed a dynamic stiffness method for the analysis of a simply supported stiffened shell. The stiffeners are considered as smeared over the surface of the structures and is presumed to be sufficient for describing the lower modes of vibration of the stiffened structures. It permits deriving appropriate modifications of the shell differential equations. These equations are then solved by considering the shell as simply supported, yielding the dynamic stiffness matrix. This approach is dedicated to analysing aircraft fuselages. The shell is in vacuo (i.e. not coupled with an internal or external fluid), making the approach interesting for analysing shell behaviour in a light fluid only. Previously, on an equivalent smeared approach, Hoppmann [10] developed and validated experimentally a technique to model the effect of stiffeners on the flexural vibrations of cylindrical shells. Its approach considers an orthotropic shell having the equivalent stiffness characteristic of a shell with closely spaced identical stiffeners.

Laulagnet and Guyader [11] analysed the characteristics of the sound radiated by a finite ringstiffened cylindrical shell submerged in a fluid by using a modal approach. The approach is valid in a heavy fluid like water. The difficulty of estimating the modal radiation impedances numerically restricts this method to low frequencies. Yan et al. [12,13] applied the space harmonic approach to predict the sound radiation from a submerged periodic ring-stiffened shell. By using the Bloch-Floquet theorem, the response of the periodic structure to harmonic excitations has been obtained by expanding it in the terms of a series of space harmonics. The radiated sound power of the shell and the influence of different parameters have been studied by 
this approach for low circumferential mode orders. Comparisons were performed with the power flow propagation in the equivalent unstiffened shell. Prior to this and with a similar approach, Hodges et al. [14] analysed in detail the dynamic behaviour of a periodic ribbed cylinder in vacuo in the low frequency range. Good agreement was obtained between their model and the measurements of a ribbed cylinder over a frequency range from zero to about three times the ring frequency. The edges of the pass bands as a function of circumferential wavenumber were especially well predicted. Zhang et al. [15] developed a hybrid method based on an energy finite element formulation to predict the high-frequency vibration response of fluid loaded cylindrical shells with periodic circumferential stiffeners. Added mass and radiation effects related to the surrounding exterior fluid medium were taken into account, but the radiated pressure in the fluid was not estimated. Photiadis et al. have published numerical and experimental results concerning scattering from a ribbed cylindrical shell [16-21]. The model of an infinite ribbed cylindrical shell is based on the Bloch-Floquet theorem in [16]. They also analysed the effect of an irregularly ribbed fluid loaded cylindrical shell using an axisymmetric finite element / infinite element model [20]. Calculations were achieved for a frequency range up to three times the ring frequency. Their numerical results were in good agreement with the experiments and localised the vibration related to the rib aperiodicity. However, this axisymmetric finite element / infinite element model is limited to low frequencies, as the higher the frequency the greater the number of degrees of freedom.

Acoustic radiation from an infinite fluid-loaded, laminated composite shell, which is reinforced by doubly periodic rings, was investigated by Yin et. al. [22]. The rings interact with the shell only through normal forces. The solution for the radial displacement in the wave number domain is obtained by using the method developed by Mace [7] for an infinite flat plate. 
The scattering of sound waves by a periodically ribbed shell was also studied by Tran-Van-Nhieu [23]. He considered a shell of finite length that differs from most publications dealing with infinite cylindrical shells. The theoretical formulation of his model is based on an adaptation of the space-harmonic series usually used for the infinite shell. The expansion of the solution near the shell is performed on the shape functions as defined by Miles [24] for finite periodic simply supported structures. In these developments, only the normal component of the reactive force applied by the ring to the shell is considered. The author emphasizes that this assumption is rather restrictive as the other components of the reactive forces might considerably change the result. Comparisons of this model with experiments are proposed by Liétard et al. [25]. The model allows highlighting the physical phenomena involved in scattering waves on the finite ribbed shell in monostatic configuration.

Recently, Caresta et al. [26,27] studie the low frequency vibrational behaviour and radiated sound of a submarine hull under axial excitation. The submarine is modelled as a fluid-loaded cylindrical shell with internal bulkheads and ring-stiffeners and closed at each end by truncated conical shell. The stiffeners are modelled using 1D beam model and a smeared approach whereas the bulkheads are modelled as thin circular plates in bending and in-plane motion. The computational results were validated by comparing with finite element and boundary element results. This model permits to study the influence of the various complicating effects such as the bulkheads, ring-stiffened and fluid loading on the structural and acoustic responses of the shell. In the context of the active control of low-frequency radiated pressure, a simplified model of a submarine hull under axial excitation was developed by Pan et al. [28]. A water-loaded finite stiffened cylindrical shell with rigid ends caps is considered. This model permits to examine the effects of control actions, both structurally and acoustically, for a control moment applied around the circumference of the hull in the low frequencies. 
In the area of the discretization method, a finite-element structural-acoustic modelling technique has been presented [29] to analyse the axial symmetric geometries subject to nonsymmetric loading. Scattering or radiation of stiffened shells can be studied using this technique. The main feature of the model is the use of the Bérenger Perfectly Matched Layer (PML) to emulate the Sommerfeld radiation condition. The advantage of PML is that an FE mesh of minimal size is necessary to represent the fluid domain, taking the free field condition into account. The proposed technique is validated by comparison with analytical solutions and with other numerical models. However, it is difficult to appreciate the gain of this approach in term of computing times compared to classical infinite elements and boundary elements methods.

Little attention has been devoted in the past to modelling stiffeners in the stiffened shell. It is generally assumed that the stiffeners behave like beams. This assumption is valid in the low frequency domain but finite element calculations for a typical submarine stiffener have shown than deformation of the cross section can be observed at about $10 \mathrm{~Hz}$. The behaviour of the stiffeners can strongly influence the vibration of the shell and its acoustic radiation. Accurate modelling of the internal frames of the shell should be taken into account in the stiffened shell model. This point will be addressed in particular in this paper.

The model developed here permits considering a shell immerged in a fluid and stiffened by the ring stiffeners and the axisymmetric bulkheads of a submarine pressure hull. The ring stiffeners can have different spacings and different cross-sections. The cylindrical shell has an infinite length although finite length can be simulated by introducing boundary conditions on dummy internal frames. In this case, the left-over shell outside the dummy internal frames (i.e. boundary 
conditions) has a role of a non rigid cylindrical baffle. The approach will be described by considering the shell or the frames excited by a mechanical point force. It is not a restriction of the approach. It can be easily extended to other sorts of excitation such as external plane waves for scattering studies.

In the next section, the principle of the Circumferential Admittance Approach (CAA) is presented. This permits assembling a model of the fluid loaded shell with finite element models of the internal frames. The FE estimation of the circumferential admittances of the frames is given in Sec. 3, whereas three methods are proposed in Sec. 4 to estimate the circumferential admittances of the fluid loaded shell. An overview of the approach is then given in Sec. 5. The final results are the spatial distributions of the vibratory field and the radiated pressure field by the shell coupled with its internal frames. Sec. 6 is dedicated to comparing the present approach with other numerical results. In particular, the accuracy of the approach is studied as a function of the three methods described in Sec. 4 to estimate the shell admittances. It permits defining an optimal approach. An example of a submarine application is then proposed to illustrate the efficiency of the present approach.

\section{THE PRINCIPLE OF THE CIRCUMFERENTIAL ADMITTANCE APPROACH}

A ring-stiffened cylindrical shell immersed in water shown on Fig. 1a is considered. It is characterized by constant thickness $h$, mean radius $R$, mass density $\rho$, Young modulus $E$, and Poisson ratio $v$. The shell is assumed to have an infinite length but clamped boundary conditions could be introduced later to simulate the finite length of a submarine hull. We consider the cylindrical coordinates $(r, \theta, x)$ where $r$ is the radial coordinate, $\theta$ the circumferential angular coordinate and $x$ the axial coordinate, as shown on Fig 1a. The external fluid has mass density $\rho_{0}$ 
and acoustic wave speed $c_{0}$. The $\Theta$ internal frames are composed of different types of stiffeners, bulkheads and hemispherical endcaps. Theses frames are assumed to be axially symmetric and the junctions with the shell are located at axial coordinate $x_{i}, i \in[1, \Theta]$. For example, the stiffeners could have different T-shaped cross sections, the bulkheads and the end caps could be curved circular plates. The materials of the shell and frames are linearly elastic, homogeneous and isotropic. Moreover, we assume that the motions of the shell and the frames are described by thin shell theories. Then, the displacements are expressed at points on the mid-surface of the shell models. The connections between the shell and the frames are rigid, so that, at each line of attachment, the shell and the frame have the same linear velocity and angular velocity. To shorten the presentation, the external load is assumed to be a mechanical point force acting in the plane $\theta=0$. However, it is not a limitation of the technique which may be easily extended to more complicated excitations. This excitation is harmonic with time dependence $e^{j \omega t}$ where $\omega$ is the angular frequency. We are interested in the stationary response of the framed shell. Then, in the following, dependence $e^{j \omega t}$ for the excitation and the response will be omitted in the notation although it is always considered.

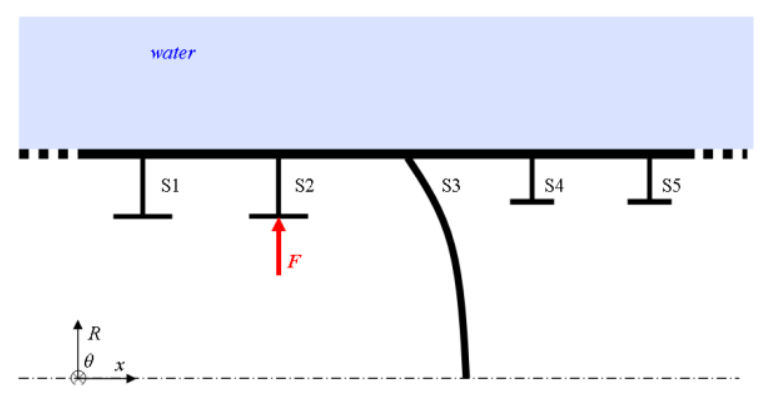

(a)
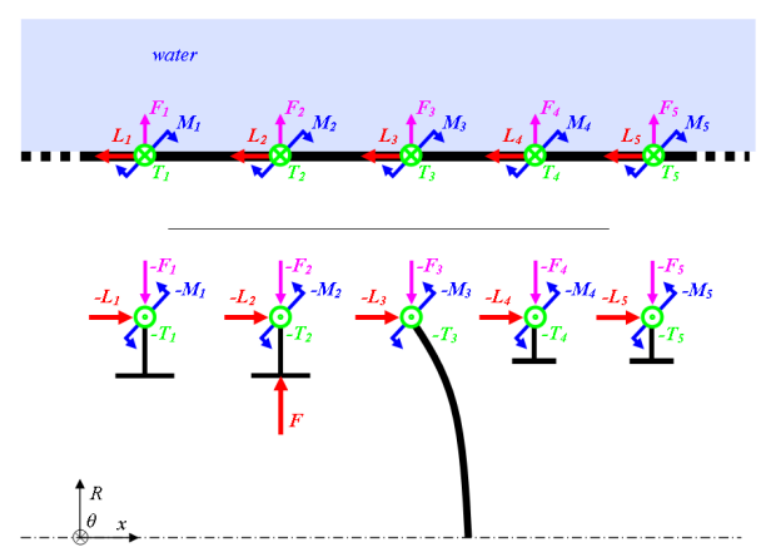

(b) 
FIG. 1. (a), Illustration of the framed shell immerged in water ; (b), Partitioning and coupling forces.

As shown in Fig. 1b, the system considered is partitioned such that the fluid loaded shell constitutes one subsystem and the frames constitute other independent subsystems. The following is defined at the junction between the shell and the $i^{\text {th }}$ frame:

- $W_{i}^{\text {shell }}, V_{i}^{\text {shell }}, U_{i}^{\text {shell }}$, and $\varphi_{i}^{\text {shell }}$ (respectively $W_{i}^{\text {frame }}, V_{i}^{\text {frame }}, U_{i}^{\text {frame }}$ and $\varphi_{i}^{\text {frame }}$ ) the radial / tangential / axial displacements, and the angular rotation $(\varphi=\partial W / \partial x)$ of the shell (respectively the frame);

- $F_{i}^{\text {shell }}, T_{i}^{\text {shell }}, L_{i}^{\text {shell }}$ and $M_{i}^{\text {shell }}$ (respectively $F_{i}^{\text {frame }}, T_{i}^{\text {frame }}, L_{i}^{\text {frame }}$ and $M_{i}^{\text {frame }}$ ) the radial / tangential / axial forces, and the angular moment acting on the frame (respectively shell) by the shell (respectively frame).

The circumferential amplitudes of these quantities are defined by their Fourier transforms about coordinate $\theta$ :

$$
\begin{aligned}
& \mathfrak{R} \longrightarrow \mathfrak{R} \\
& f(\theta) \stackrel{F . T .}{\longrightarrow} \tilde{f}\left(k_{\theta}\right)=\int_{\Re} f(\theta) e^{-j k_{\theta} \theta} d \theta
\end{aligned}
$$

where $f$ is the considered quantity and $k_{\theta}$ is the circumferential wave number.

$\tilde{f}\left(k_{\theta}\right)$ indicates the Fourier transform about the circumference of $f(\theta)$.

$\theta=0$ is a symmetric plane of the considered system (i.e. the stiffened shell excited by the force contained in the plane $\theta=0)$. Taking periodicity $2 \pi$ about the circumference into account, the properties of the Fourier transform give:

$$
\left\{\begin{array}{l}
\left|\tilde{f}\left(-k_{\theta}\right)\right|=\left|\tilde{f}\left(k_{\theta}\right)\right| \\
\tilde{f}\left(k_{\theta}\right)=0, \text { if } k_{\theta} \text { is not an integer. }
\end{array}\right.
$$


In accordance with these properties, the calculations are performed in the following for a circumferential wave number $k_{\theta}$ equal to a natural number called the circumferential order, $n$ $\left(k_{\theta}=n\right)$. It should be noted that another possible approach is to expand the quantity $f(\theta)$ with a Fourier series. In the rest of this paper, this circumferential wave number will be omitted in the notation.

In practice, the summation about $n$ to obtain $f(\theta)$ from $\tilde{f}\left(k_{\theta}\right)$ is truncated at a maximal circumferential order $\bar{N}(n \in[0, \bar{N}])$. The criterion for defining $\bar{N}$ will be discussed later (Sec. $5)$.

We define:

- the circumferential admittances of the shell (respectively frame) between the $i^{\text {th }}$ junction and the $j^{\text {th }}$ junction, $\tilde{Y}_{\xi_{i} \zeta_{j}}^{\text {shell }}\left(\right.$ respectively $\left.\tilde{Y}_{\xi_{i} \zeta_{j}}^{\text {frame }}\right)$ by:

$$
\tilde{Y}_{\xi_{i} \zeta_{j}}^{\text {shell }}=\frac{\tilde{\xi}_{i}^{\text {shell }}}{\tilde{\zeta}_{i}^{\text {shell }}}\left(\operatorname{resp} . \tilde{Y}_{\xi_{i} \zeta_{j}}^{\text {frame }}=\frac{\tilde{\xi}_{i}^{\text {frame }}}{\tilde{\zeta}_{i}^{\text {frame }}}\right)
$$

where $\xi \in\{W, U, V, \varphi\}$ and $\zeta \in\{F, L, T, M\}$.

- the free circumferential displacement of the shell, $\tilde{\bar{\xi}}_{i}^{\text {shell }}$ (respectively frame, $\tilde{\bar{\xi}}_{i}^{\text {frame }}$ ) as the circumferential displacements of the uncoupled shell (respectively frame) excited by the external load.

These circumferential admittances and free displacements are evaluated on each uncoupled subsystem. For example, if the shell is only excited on the $I^{\text {th }}$ frame, all the free circumferential displacements are null except for the $I^{\text {th }}$ frame: 


$$
\left\{\begin{array}{l}
\tilde{\bar{\xi}}_{i}^{\text {shell }}=0, \forall i \in[1, \Theta] \\
\tilde{\xi}_{i}^{\text {frame }}=0, \forall i \in\{[1, \Theta] / i \neq I\} \text { and } \tilde{\xi}_{I}^{\text {frame }}=\hat{\tilde{\xi}}
\end{array}\right.
$$

Using the superposition principle for a linear passive system, displacement continuity and equilibrium conditions at the junctions between the shell and the frames, we obtain a linear equation system which can be written in matrix form:

$$
\left[\tilde{\mathbf{Y}}^{\text {shell }}+\tilde{\mathbf{Y}}^{\text {frame }}\right] \tilde{\mathbf{F}}^{\text {frame }}=\tilde{\mathbf{W}}^{\text {frame }}-\tilde{\mathbf{W}}^{\text {shell }}
$$

where the $4 \Theta \times 4 \Theta$ matrices, $\tilde{\mathbf{Y}}^{\text {shell }}$ and $\tilde{\mathbf{Y}}^{\text {frame }}$, are given by:

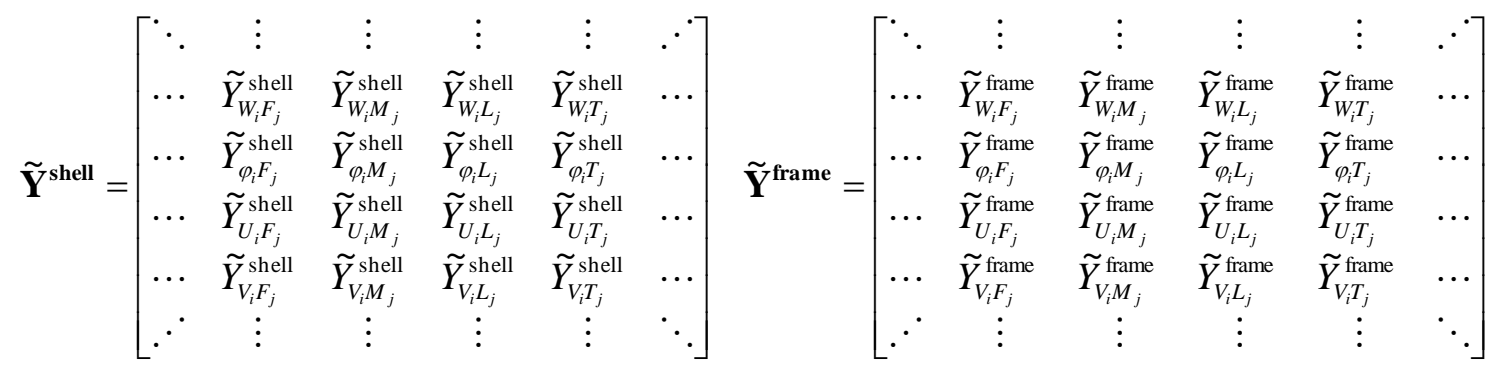

and, the $4 \Theta$-dimensional vectors $\tilde{\mathbf{F}}^{\text {frame }}, \tilde{\bar{W}}^{\text {shell }}, \tilde{\bar{W}}^{\text {frame }}$ are:

$$
\tilde{\mathbf{F}}^{\text {frame }}=\left[\begin{array}{c}
\vdots \\
\tilde{F}_{j}^{\text {frame }} \\
\tilde{M}_{j}^{\text {frame }} \\
\tilde{L}_{j}^{\text {frame }} \\
\tilde{T}_{j}^{\text {frame }} \\
\vdots
\end{array}\right] \quad \tilde{\bar{W}}^{\text {shell }}=\left[\begin{array}{c}
\vdots \\
\tilde{\bar{W}}_{j}^{\text {shell }} \\
\tilde{\bar{\varphi}}_{j}^{\text {shell }} \\
\tilde{\bar{U}}_{j}^{\text {shell }} \\
\tilde{\bar{V}}_{j}^{\text {shell }} \\
\vdots
\end{array}\right] \quad \tilde{\bar{W}}^{\text {frame }}=\left[\begin{array}{c}
\vdots \\
\tilde{\bar{W}}_{j}^{\text {frame }} \\
\tilde{\bar{\varphi}}_{j}^{\text {frame }} \\
\tilde{\bar{U}}_{j}^{\text {frame }} \\
\tilde{\bar{V}}_{j}^{\text {frame }} \\
\vdots
\end{array}\right]
$$

By solving this system having $4 \Theta$ unknowns, we deduce the forces and the moments exerted by the frames on the shell when they are coupled together. In the second step these reaction forces and moments are injected in a shell model to deduce the vibration and the radiated pressure of the shell coupled with its internal frames. Before doing this, we propose to describe the process to 
estimate $\tilde{\mathbf{Y}}^{\text {frame }}, \tilde{\overline{\mathbf{W}}}^{\text {frame }}$ in Sec. 3 and $\tilde{\mathbf{Y}}^{\text {shell }}, \tilde{\mathbf{W}}^{\text {shell }}$ in Sec. 4. These quantities are evaluated on each uncoupled subsystem, independently of each other, which is one of the main advantages of this approach.

\section{ESTIMATION OF FRAMES ADMITTANCES}

The shell theory is used to describe the dynamic behaviour of each type of frame: stiffeners, bulkheads and endcaps. Axisymmetric Finite Elements can be used to estimate the circumferential admittances and free displacements of these frames. In this paper, the calculations are performed with the MSC/NASTRAN code [30]. The conical shell elements CCONEAX with the PCONEAX properties permit describing the frame behaviour from a mesh of the cross section. The classical criterion which consists in dividing by 6 the smallest natural wavelength is used to determine the length of the elements. The complex geometry of the cross section can be described and thickness variations can be taken into account easily. The calculations are performed for harmonic orders of Fourier series (AXIS entry in NASTRAN) that are equivalent to the circumferential orders of the present paper. Displacements at the junction are evaluated for the four harmonic loads ( 3 forces and 1 moment) using a direct frequency response analysis [30] (solution sequence SOL 108). For each harmonic order, NASTRAN solves numerically the matrix system of the axisymmetric finite element. The calculation times are reasonable due to the use of the axisymmetric property of the frames.

Pre and post-processing are done using a MATLAB code. It should be noted that these calculations are necessary for each type of frame, but not for all the frames, thereby lightening the process. An example of Finite Elements results is proposed in the next two figures. The stiffener considered has the typical mechanical and geometrical characteristics of a submarine pressure hull stiffener. The mesh of the $\mathrm{T}$ cross section is made of 12 nodes (RINGAX) and 12 
elements (CCONEAX). The simulation results in Fig. 2 illustrates the deformations of the crosssection of this stiffener at $1000 \mathrm{~Hz}$ for the circumferential order $n=0$. These deformations contradict the classical 1D beam assumptions (i.e. Euler-Bernoulli assumptions) and explain the differences observed in Fig. 3 that compares the plots of the circumferential admittances $\tilde{Y}_{\theta M}$ calculated with NASTRAN (i.e. FEM results) and with the analytical 1D beam model (see [7]). The 1D beam formulae do not permit the correct estimation of the admittances of this stiffener, especially for the torsional motions (the differences for $\tilde{Y}_{W F}$ are less significant). This is an example that led us to model the stiffener behaviour by using shell theory and the Finite Elements Method.

$1000 \mathrm{~Hz}$ - Circonf. arder : Q - Moment excit ation

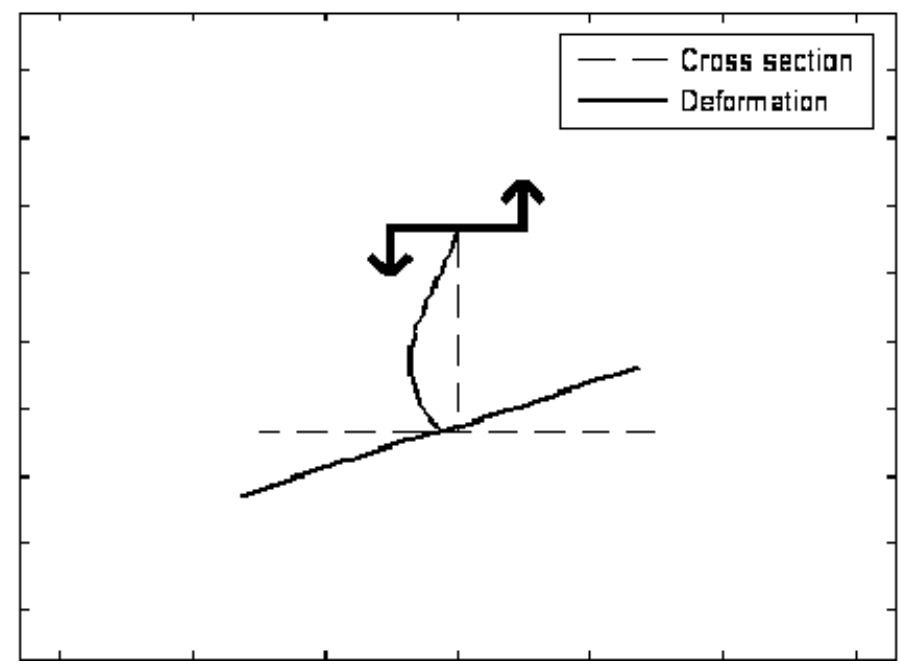

FIG. 2. Deformation of a stiffener cross-section for a moment excitation. $1000 \mathrm{~Hz}, \mathrm{n}=0$.

T section: $300 \mathrm{~mm}$ x $60 \mathrm{~mm} / 60 \mathrm{~mm}$ x $300 \mathrm{~mm}$. R=5 m. Material: steel $(\eta=0.005)$. 


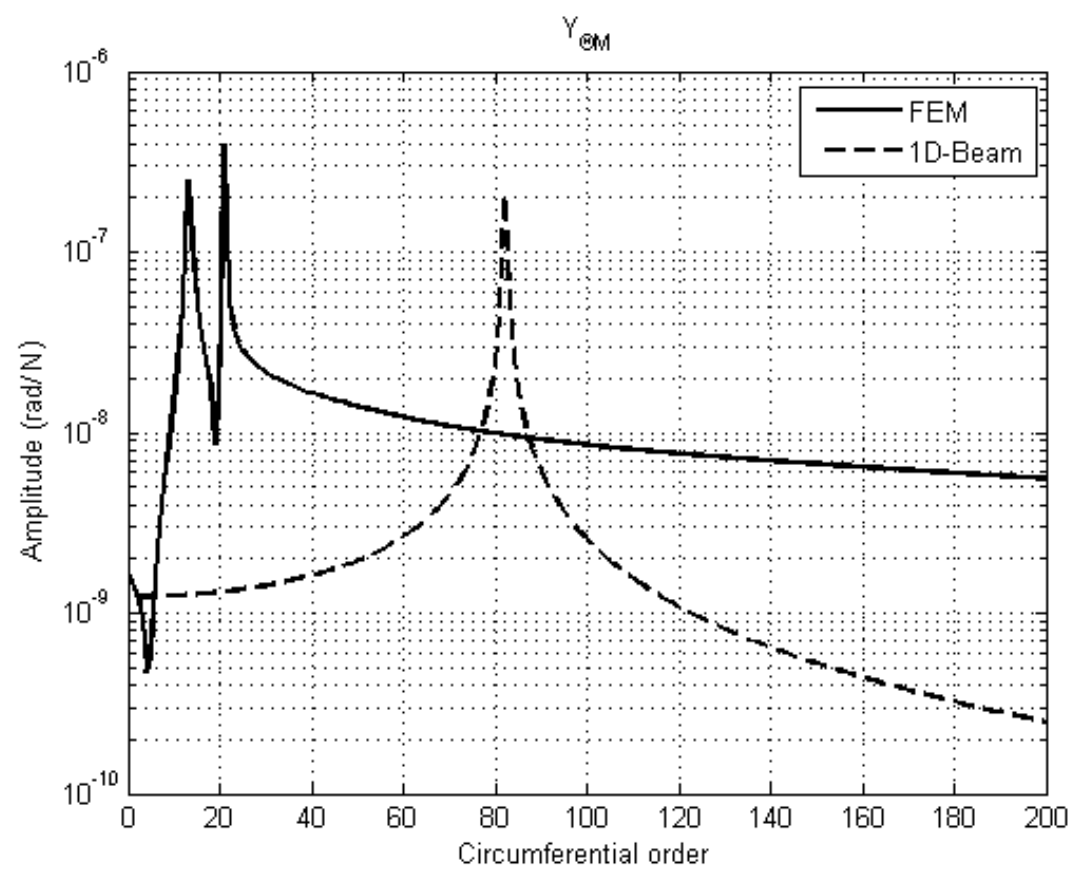

FIG. 3. Comparison of the circumferential admittances $\tilde{Y}_{\theta M}^{\text {frame }}$ calculated with NASTRAN (full line) and with the $1 \mathrm{D}$-beam model (dash line). $1000 \mathrm{~Hz}\left(\mathrm{~dB}\right.$ ref. $\left.1 \mathrm{~N}^{-1}\right)$.

Concerning the frame admittances, it should underlined that a finite length of the shell could be emulated by introducing a fictive frame at the assumed ends of the shell and by attributing a null circumferential admittance at its frame. In this case, the fictive frame will introduce clamped boundary conditions.

\section{ESTIMATION OF SHELL ADMITTANCES}

In this section, we describe three methods of estimating the circumferential admittances of the fluid loaded shell. The first is a numeric approach based on the wave-number formulation of the problem and the use of an inverse discrete Fourier transform. The second consists of solving 
analytically a simplified problem, whereas the last method consists in combining the two previous methods. The advantages and drawbacks of these methods will be discussed in Sec. 6 .

We emphasize that an infinite cylindrical shell is considered to estimate the shell admittance but the approach presented in this paper is not restricted for simulating the behaviour of infinite shell. Indeed, finite length of the shell can be simulated with CAA by introducing boundary conditions on dummy internal frames. For example, clamped boundary conditions at the ends of the shell could be introduced with dummy frames having null circumferential admittances. The left-over shell outside the two dummy frames has then a role of a non rigid cylindrical baffle.

\subsection{PRESENTATION OF THE FLUID LOADED SHELL PROBLEM}

Here we present the problem considered to estimate shell admittance. It is composed of a cylindrical shell of infinite length immerged in fluid. As shown in Fig. 4, the shell is excited by a line load $\zeta$ on the circumference at $x=0$. The direction of $\zeta$ will be defined later as a function of the definition of the admittances being estimated. The circumferential amplitudes of this load are assumed to be unitary $(\tilde{\zeta}=1)$. The admittances between two junctions will be deduced by translating the circumferential displacement of this shell $\tilde{\xi}$ along $x$. Indeed, the shell admittances between the $i^{\text {th }}$ junction and the $j^{\text {th }}$ junction $\tilde{Y}_{\xi_{i} \zeta_{j}}^{\text {shell }}$ could be deduced by using the relation:

$$
\tilde{Y}_{\xi_{i} \zeta_{j}}^{\text {shell }}=\tilde{\xi}_{\zeta}\left(x_{i}-x_{j}, k_{\theta}\right), \forall(i, j) \in([1, \Theta])^{2} .
$$

It should be recalled that $U, V$, and, $W$ are respectively the axial, tangential and radial displacements of the shell whereas $L, T$, and, $F$ are respectively the axial, tangential and radial forces exerted on the shell by the external load. 


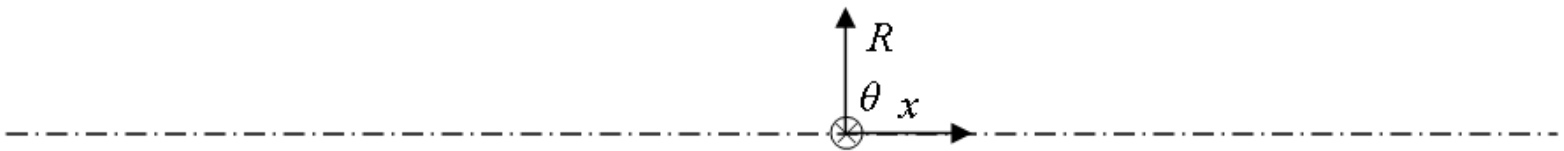

FIG. 4. Infinite cylindrical shell immersed in water and excited by line excitations at $x=0$.

The dynamic behaviour of this shell can be described by the Flugge equations of motions [31,32]. The external forces and the parietal pressure appear in the second member of these equations:

$$
\begin{aligned}
& {\left[R^{2} \frac{\partial^{2}}{\partial x^{2}}+\frac{1-v}{2} \frac{\partial^{2}}{\partial \theta^{2}}-\rho R^{2} \frac{1-v^{2}}{E} \frac{\partial^{2}}{\partial t^{2}}+\beta^{2} \frac{1-v}{2} \frac{\partial^{2}}{\partial \theta^{2}}\right] U} \\
& +\left[R \frac{1+v}{2} \frac{\partial^{2}}{\partial x \partial \theta}\right] V+\left[R v \frac{\partial}{\partial x}-\beta^{2} R^{3} \frac{\partial^{3}}{\partial x^{3}}+\beta^{2} R \frac{1-v}{2} \frac{\partial^{3}}{\partial x \partial \theta^{2}}\right] W \\
& =-\frac{\left(1-v^{2}\right) R^{2}}{E h} L \delta(x)
\end{aligned}
$$

$$
\begin{aligned}
& {\left[R \frac{1+v}{2} \frac{\partial^{2}}{\partial x \partial \theta}\right] U} \\
& +\left[R^{2} \frac{1-v}{2} \frac{\partial^{2}}{\partial x^{2}}+\frac{\partial^{2}}{\partial \theta^{2}}-\rho R^{2} \frac{1-v^{2}}{E} \frac{\partial^{2}}{\partial t^{2}}+3 \beta^{2} R^{2} \frac{1-v}{2} \frac{\partial^{2}}{\partial x^{2}}\right] V \\
& +\left[\frac{\partial}{\partial \theta}-\beta^{2} R^{2} \frac{3-v}{2} \frac{\partial^{3}}{\partial x^{2} \partial \theta}\right] W=-\frac{\left(1-v^{2}\right) R^{2}}{E h} T \delta(x)
\end{aligned}
$$




$$
\begin{aligned}
& {\left[R v \frac{\partial}{\partial x}-\beta^{2} R^{3} \frac{\partial^{3}}{\partial x^{3}}+\beta^{2} R \frac{1-v}{2} \frac{\partial^{3}}{\partial x \partial \theta^{2}}\right] U+\left[\frac{\partial}{\partial \theta}-\beta^{2} R^{2} \frac{3-v}{2} \frac{\partial^{3}}{\partial x^{2} \partial \theta}\right] V} \\
& +\left[1+\beta^{2}\left(R^{4} \frac{\partial^{4}}{\partial x^{4}}+2 R^{2} \frac{\partial^{4}}{\partial x^{2} \partial \theta^{2}}+\frac{\partial^{4}}{\partial \theta^{4}}\right)+\rho R^{2} \frac{1-v^{2}}{E} \frac{\partial^{2}}{\partial t^{2}}+\beta^{2}\left(1+2 \frac{\partial^{2}}{\partial \theta^{2}}\right)\right] W \\
& =\frac{\left(1-v^{2}\right) R^{2}}{E h}\left(F \delta(x)-M \delta^{\prime}(x)-p\right)
\end{aligned}
$$

where: $-\delta(x)$ and $\delta^{\prime}(x)$ are the Dirac delta distribution and its derivative, respectively;

$$
\text { - } \beta=\frac{h}{R \sqrt{12}} \text {, and } p \text { is the parietal pressure exerted by the fluid on the shell. }
$$

Structural damping of the shell can be introduced by assigning a complex value with a loss factor $\eta$ to the elastic modules of the shell material: $E^{*}=E(1+j \eta)$.

In the fluid domain, pressure $p$ respects the Helmholtz equation:

$$
\Delta p+k_{0}^{2} p=0
$$

with $\Delta=\frac{\partial^{2}}{\partial x^{2}}+\frac{\partial^{2}}{\partial r^{2}}+\frac{1}{r} \frac{\partial}{\partial r}+\frac{1}{r^{2}} \frac{\partial^{2}}{\partial \theta^{2}}$ and $k_{0}=\frac{\omega}{c_{0}}$, the acoustic wavenumber.

It is assumed that the Sommerfeld conditions and the normal velocity continuity at the interface shell/fluid are respected.

We now describe the three methods for estimating the circumferential displacements of this shell.

\subsection{METHOD 1: RESOLUTION WITH A SPECTRAL APPROACH}

The problem described previously can be solved in the wave-number domain. This consists in applying a space-Fourier transform at the Flügge equations (9a-9c). This transform is defined by: 


$$
\begin{gathered}
\mathfrak{R}^{2} \longrightarrow \mathfrak{R}^{2} \\
f(x, \theta) \stackrel{\text { S.F.T. }}{\longrightarrow} \tilde{\tilde{f}}\left(k_{x}, k_{\theta}\right)=\int_{\mathfrak{R}^{2}} f(x, \theta) e^{-j\left(k_{x} x+k_{\theta} \theta\right)} d x d \theta,
\end{gathered}
$$

where:

- $\tilde{\tilde{f}}\left(k_{x}, k_{\theta}\right)$ denotes the space Fourier transform of $f(x, \theta)$, and;

- $k_{x}, k_{\theta}$ are respectively the axial wavenumber and the circumferential wavenumber.

The Flügge equations become:

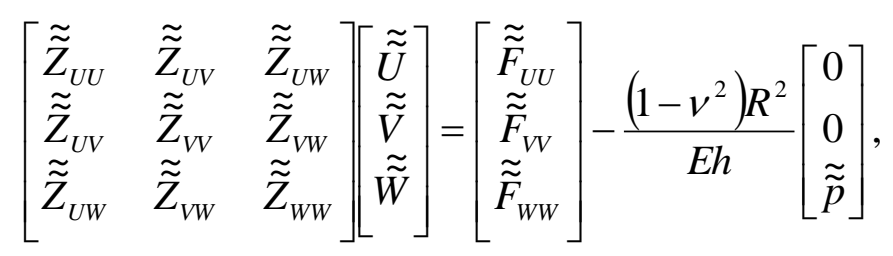

with $\tilde{\widetilde{Z}}_{U U}=-R^{2} k_{x}^{2}-k_{\theta}{ }^{2} \frac{1-v}{2}\left(1+\beta^{2}\right)+R^{2} k_{l}^{2}, \tilde{\widetilde{Z}}_{U V}=-R \frac{1+v}{2} k_{\theta} k_{x}$,

$\tilde{\widetilde{Z}}_{U W}=j k_{x}\left(R v+\beta^{2} R^{3} k_{x}^{2}-\beta^{2} R \frac{1-v}{2} k_{\theta}^{2}\right), \tilde{\widetilde{Z}}_{V V}=-R^{2} k_{x}{ }^{2} \frac{1-v}{2}\left(1+3 \beta^{2}\right)-k_{\theta}{ }^{2}+R^{2} k_{l}^{2}$,

$\tilde{\widetilde{Z}}_{V W}=j k_{\theta}\left(1+\beta^{2} R^{2} \frac{3-v}{2} k_{x}^{2}\right), \tilde{\widetilde{Z}}_{W W}=1+\beta^{2}\left[R^{4} k_{x}{ }^{4}+k_{\theta}{ }^{2}\left(2 R^{2} k_{x}{ }^{2}+2\right)+k_{\theta}{ }^{4}+1\right]-R^{2} k_{l}{ }^{2}$,

$\tilde{\widetilde{F}}_{U U}=-\frac{\left(1-v^{2}\right) R^{2}}{E h} \tilde{\tilde{L}}, \tilde{\widetilde{F}}_{V V}=-\frac{\left(1-v^{2}\right) R^{2}}{E h} \tilde{\tilde{T}}, \tilde{\widetilde{F}}_{W W}=\frac{\left(1-v^{2}\right) R^{2}}{E h}\left(\tilde{\widetilde{F}}+j k_{x} \tilde{\tilde{M}}\right)$

where $k_{l}=\omega \sqrt{\frac{\rho\left(1-v^{2}\right)}{E}}$.

The circumference periodicity of the shell requires that the spectral displacements $\tilde{\widetilde{U}}, \tilde{\tilde{V}}, \tilde{\tilde{W}}$ differ from zero only for the circumferential wave-numbers equalling the circumferential orders $\left(k_{\theta}=n\right)$.

The spectral pressure $\tilde{\tilde{p}}$ can be evaluated by resolving the Helmholtz equation [33]: 


$$
\begin{gathered}
\tilde{\widetilde{p}}=\tilde{\bar{Z}}_{\text {fluid }} \tilde{\tilde{W}}, \\
\tilde{\bar{Z}}_{\text {fluid }}=\frac{\rho_{0} \omega^{2}}{k_{r}} \frac{H_{n}^{(2)}\left(k_{r} R\right)}{H_{n}^{(2)}\left(k_{r} R\right)},
\end{gathered}
$$

where: $-k_{r}=\sqrt{k_{0}{ }^{2}-k_{x}^{2}}$;

- $\rho_{0}$ is the fluid density, and;

- $H_{n}^{(2)}$ and $H_{n}^{\prime(2)}$ are, respectively, the Hankel functions of the second kind and order $n$, and their derivatives respect to the argument.

By introducing Eq. (16) in Eq. (14), we obtain a solvable linear equation system That provides the analytical expressions for the spectral displacements $\tilde{\tilde{W}}, \tilde{\widetilde{U}}, \tilde{\tilde{V}}$ :

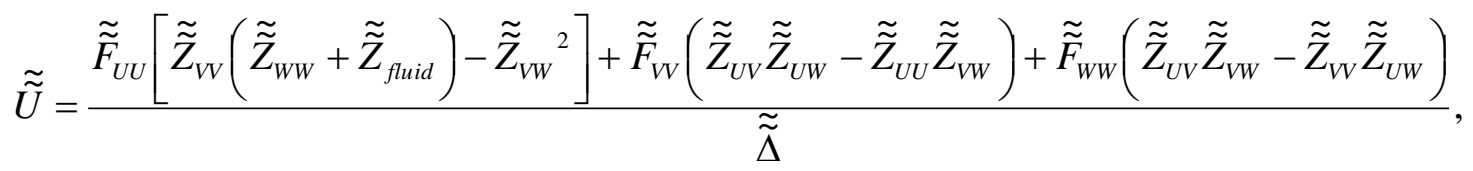

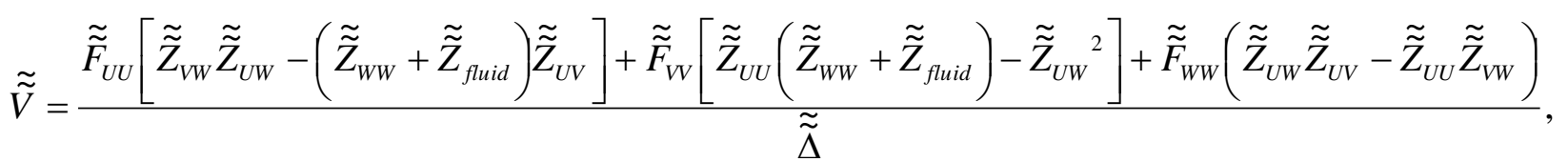

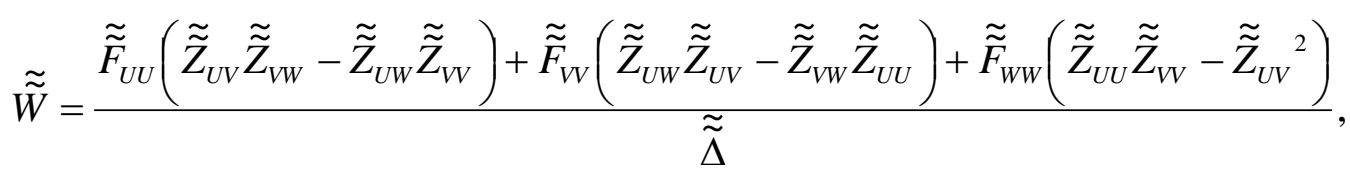

where:

$$
\begin{aligned}
& \tilde{\widetilde{\Delta}} \tilde{\widetilde{Z}}_{U W}\left(\tilde{\widetilde{Z}}_{U V} \tilde{\widetilde{Z}}_{V W}-\tilde{\widetilde{Z}}_{U W} \tilde{\widetilde{Z}}_{V V}\right)+\tilde{\widetilde{Z}}_{V W}\left(\tilde{\widetilde{Z}}_{U W} \tilde{\widetilde{Z}}_{U V}-\tilde{\widetilde{Z}}_{V W} \tilde{\widetilde{Z}}_{U U}\right)+\left(\tilde{\widetilde{Z}}_{W W}+\tilde{\widetilde{Z}}_{\text {fluid }}\right)\left(\tilde{\widetilde{Z}}_{U U} \tilde{\widetilde{Z}}_{V V}-\tilde{\widetilde{Z}}_{U V}{ }^{2}\right) \\
& \text { and } \tilde{\widetilde{Z}}_{\text {fluid }}=\frac{\left(1-v^{2}\right) R^{2}}{E h} \tilde{\bar{Z}}_{\text {fluid }}
\end{aligned}
$$


The spectral rotation $\tilde{\tilde{\varphi}}$ is deduced from the Fourier transform of $\varphi=\frac{\partial W}{\partial x}$ :

$$
\tilde{\widetilde{\varphi}}=j k_{x} \tilde{\widetilde{W}}
$$

To evaluate the admittance $\tilde{Y}_{\xi \zeta}^{\text {shell }}$ with $\xi \in[W, U, V, \varphi]$ and $\xi \in[F, L, T, M]$, we calculate the circumferential displacements $\tilde{\xi}_{\zeta}$ when the shell is excited by force $\zeta$ of unit circumferential amplitudes $(\tilde{\zeta}=1)$. The spectral forces $\tilde{\widetilde{F}}_{U U}, \tilde{\widetilde{F}}_{V V}, \tilde{\widetilde{F}}_{W W}$ are then given by:

$$
\begin{aligned}
& \text { - if } \zeta=F \Rightarrow \widetilde{\widetilde{F}}_{U U}=0, \widetilde{\widetilde{F}}_{V}=0, \widetilde{\widetilde{F}}_{W W}=\frac{\left(1-v^{2}\right) R^{2}}{E h} \text {; } \\
& \text { - if } \zeta=M \Rightarrow \tilde{\widetilde{F}}_{U U}=0, \tilde{\widetilde{F}}_{V V}=0, \widetilde{\widetilde{F}}_{W W}=j k_{x} \frac{\left(1-v^{2}\right) R^{2}}{E h} \\
& \text { - if } \zeta=L \Rightarrow \widetilde{\widetilde{F}}_{U U}=-\frac{\left(1-v^{2}\right) R^{2}}{E h}, \widetilde{\widetilde{F}}_{V V}=0, \widetilde{\widetilde{F}}_{W W}=0 \\
& \text { - if } \zeta=T \Rightarrow \widetilde{\widetilde{F}}_{U U}=0, \widetilde{\widetilde{F}}_{V V}=-\frac{\left(1-v^{2}\right) R^{2}}{E h}, \tilde{\widetilde{F}}_{W W}=0
\end{aligned}
$$

The circumferential displacements $\tilde{\xi}_{\zeta}$ can be deduced from the spectral displacement $\tilde{\xi}_{\zeta}$ by using an inverse discrete Fourier Transform about $k_{x}$ :

$$
\widetilde{\tilde{\xi}}_{\zeta}\left(k_{x}, k_{\theta}\right) \stackrel{\text { I.D.F.T. }}{\longrightarrow} \tilde{\xi}_{\zeta}\left(x, k_{\theta}\right)
$$

This transform requires truncating the wavenumber domain and sampling these wave-number fields with adequate criteria to avoid aliasing and loss of information.

This approach and the criteria were developed in reference [34] for the analysis of a stiffened plate. In the present case, the sampling wavenumber $\bar{k}_{x}$ (i.e. the truncating number about $k_{x}$ ) 
should be greater than the natural wavenumbers of the different types of motions of the shell. An illustration of the spectral displacements is given in Fig. 5. The sampling wavenumber ensures that the area of the highest displacement amplitudes is correctly taken into account.

However, for a fluid loaded cylindrical shell, the natural wavenumbers are not given by simple formulae. To overcome this obstacle, one proposes to use the same principle as the one used in FEM (finite element method) to define criteria like element lengths. The purpose of this principle is to consider each subsystem individually (i.e. part of the system), to estimate the criterion for each subsystem and then to apply the most restrictive of these criterions to the whole system. For example, to define the element length of the mesh of a structure composed of different subsystems, one generally refers to the natural wavelength of each type of motions for each uncoupled subsystem. Element length criterion for each subsystem consists then in dividing by 6 or 10 their lower natural wavelengths. This reasoning permits to have an order of magnitude of the natural wavelength of the coupled subsystems. One uses here this principle to estimate an order of magnitude for the natural wavenumbers of the fluid loaded shell. Then, one considers independently the longitudinal motions, the shear motions and the flexural motions of the shell (i.e. curvature effect neglected). 


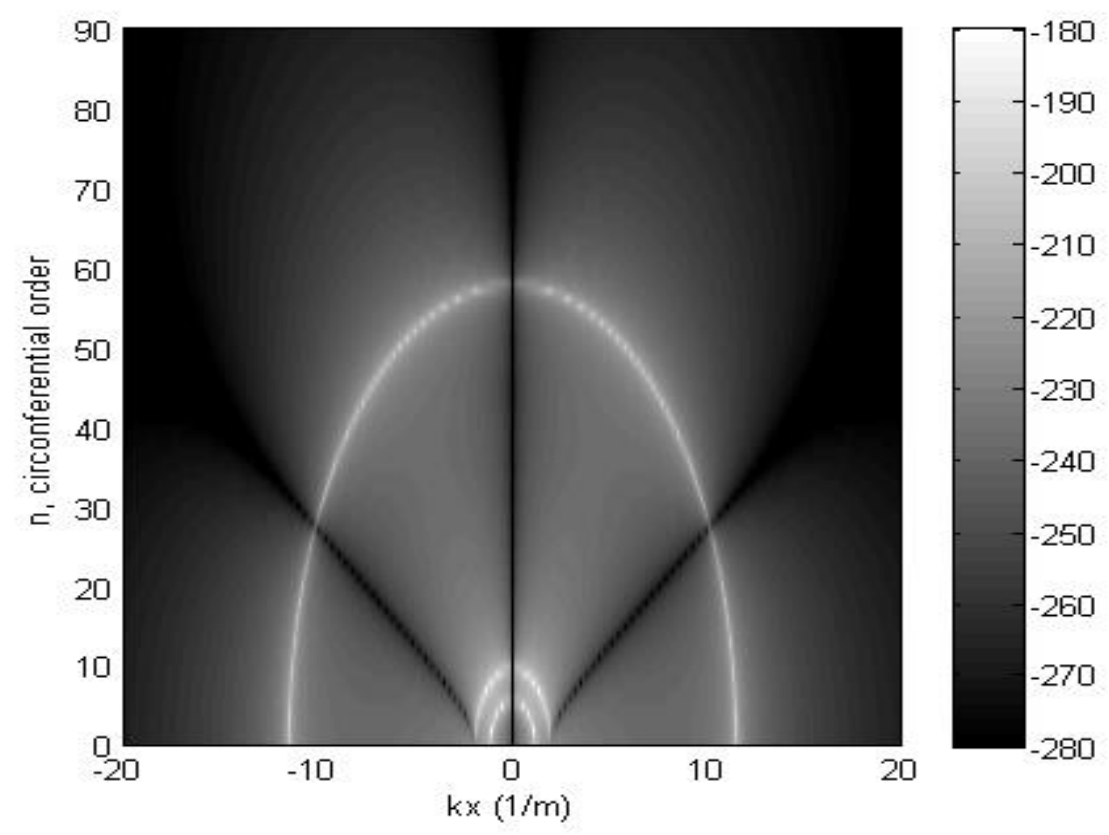

FIG. 5. Example of the radial displacement level $\left(\mathrm{dB}\right.$, ref. $\left.1 \mathrm{~m}^{2} \cdot \mathrm{N}^{-1}\right)$ in the wavenumber domain. Steel shell of $5 \mathrm{~m}$ radius and $30 \mathrm{~mm}$ thick immerged in water $(\eta=0.005)$. Case of an axial load.

The natural wavenumber for the longitudinal motions $k_{l}$ is given by expression (14) whereas the natural wavenumbers for the shear motions [35] and flexural motions are given respectively by: $k_{t}=k_{l} \sqrt{\frac{2}{1-v}}$ and $k_{f}=\sqrt{\frac{\sqrt{12}}{h} k_{l}}$. For the case of Fig. 5, one has $k_{l}=1.16 \mathrm{~m}^{-1}, k_{t}=1.95 \mathrm{~m}^{-1}$, and $k_{f}=12.48 \mathrm{~m}^{-1}$. One observes that these values permit well to localize approximately the area of the higher displacement amplitudes in the wavenumber domain.

As a thin shell is considered, we have generally:

$$
\max \left\lfloor k_{l}, k_{t}, k_{f}\right\rfloor=k_{f} \text {. }
$$

The sampling wavenumber $\bar{k}_{x}$ is therefore: 


$$
\bar{k}_{x}=\kappa_{x} \max \left[k_{f}, k_{0}\right\rfloor,
$$

where $k_{0}$ is the acoustic wavenumber and $\kappa_{x}$ is a margin coefficient (by default, we use $\kappa_{x}=2$ ).

The wavenumber resolution $\delta k_{x}$ is obtained by dividing the smallest half power bandwidths of the different natural waves by a number of $K$ points:

$$
\delta k_{x}=\frac{\Delta_{\min }}{K} \text { with } \Delta_{\min }=\min \left[\Delta_{l}^{-3 d B}, \Delta_{t}^{-3 d B}, \Delta_{f}^{-3 d B}\right],
$$

where $\Delta_{l}^{-3 d B}, \Delta_{t}^{-3 d B}, \Delta_{f}^{-3 d B}$ are the half power bandwidths for the longitudinal, torsional and flexural motions, respectively. $K$ is chosen as equal to 2 in the present paper.

The discrete Fourier transform of $\tilde{\xi}$ with this wavenumber resolution gives $\tilde{\xi}$ about $x$-axis on the space $\left[-x_{\lim }, x_{\lim }\right]$ with $x_{\lim }=\frac{\pi}{\delta k_{x}}$. A poor estimation of $\tilde{\xi}$ at the boundaries of this space can be observed due to the aliasing phenomenon. As proposed in [34], a space $[-\bar{x}, \bar{x}]$ where the effect of the aliasing can be assumed negligible can be estimated from the analysis of the energy decay of each type of wave. Equation (47) of [34] adapted to the present case gives:

$$
\bar{x}=x_{\lim }-\frac{\ln (40)}{\Delta_{\min }} .
$$

Then, the circumferential displacements $\tilde{\xi}_{\zeta}\left(x, k_{\theta}\right)$ may be used to estimate the admittances with Eq. (8). However, it is necessary to verify that the distance between the two furthermost junctions is less than the limit $\bar{x}$ :

$$
x_{\Theta}-x_{1}<\bar{x} .
$$

If this criterion is not respected, the wavenumber resolution $\delta k_{x}$ can be decreased. 
In order to limit the number of calculations, reciprocity relations can be used:

$$
Y_{U F}^{\text {shell }}=Y_{W L}^{\text {shell }}, Y_{U T}^{\text {shell }}=Y_{V L}^{\text {shell }}, Y_{V F}^{\text {shell }}=Y_{W T}^{\text {shell }}, Y_{\varphi F}^{\text {shell }}=Y_{W M}^{\text {shell }}, Y_{\varphi L}^{\text {shell }}=Y_{U M}^{\text {shell }}, Y_{\varphi T}^{\text {shell }}=Y_{V M}^{\text {shell }} .
$$

\subsection{ANALYTICAL RESOLUTION WITH SIMPLIFYING ASSUMPTIONS}

We now propose to simplify the problem and analytically calculate the shell admittances associated with the radial direction and the angular rotation (i.e. $\tilde{Y}_{W_{i} F_{j}}^{\text {shell }}, \tilde{Y}_{\varphi_{i} F_{j}}^{\text {shell }}, \tilde{Y}_{W_{i} M_{j}}^{\text {shell }}, \tilde{Y}_{\varphi_{i} M_{j}}^{\text {shell }}$ ). We therefore assume that all the other shell admittances may be neglected. Above the ring frequency, this assumption is satisfactory if the internal frames do not introduce significant couplings between the axial/torsional waves and the flexural waves of the shell coupled with its frames.

To calculate these admittances, we consider the shell excited at $x=0$ by a radial force $F$ and a moment $M$ (only) for frequencies above the ring frequency of the shell. The effects of the curvature of the shell and the couplings between the axial/torsional waves and the flexural waves may be neglected. The third equation of the Flügge model is replaced by the Love-Kircchoff equation:

$$
D^{*}\left[\frac{\partial^{4}}{\partial x^{4}}+2 \frac{\partial^{4}}{R^{2} \partial x^{2} \partial \theta^{2}}+\frac{\partial^{4}}{R^{4} \partial \theta^{4}}\right] W-\omega^{2} \rho h W=F \delta(x)-M \delta^{\prime}(x)-p
$$

where $D^{*}$ is the complex flexural rigidity given by : $D^{*}=\frac{E^{*} h R^{2}}{1-v^{2}} \beta^{2}$.

Moreover, it can be assumed that the fluid may be represented by its added mass effect on the shell, which implies that the considered frequency is well below the critical frequency of the shell. The equivalent mass density of the shell $\rho_{e}$ taking the added mass by the fluid into account 
may be approximated by the equivalent mass density of a fluid loaded plate [35] having the same thickness and material as the shell:

$$
\rho_{e}=\rho+\frac{\rho_{0}}{h \sqrt{k_{f, \text { water }}^{2}-k_{0}^{2}}}
$$

where $k_{f, w a t e r}$ is the natural flexural wavenumber of the fluid loaded plate obtained numerically by solving its dispersion relation iteratively:

$$
D k_{f, \text { water }}^{4}-\omega^{2} \rho h-\frac{\rho_{0} \omega^{2}}{h \sqrt{k_{f, \text { water }}{ }^{2}-k_{0}^{2}}}=0 .
$$

With this assumption, equation (31) becomes:

$$
D\left[\frac{\partial^{4}}{\partial x^{4}}+2 \frac{\partial^{4}}{R^{2} \partial x^{2} \partial \theta^{2}}+\frac{\partial^{4}}{R^{4} \partial \theta^{4}}\right] W-\omega^{2} \rho_{e} h W=F \delta(x)-M \delta^{\prime}(x) .
$$

We solve this problem by using the forced wave decomposition and by substructuring the shell in two parts: $\mathrm{x}<0$ and $\mathrm{x}>0$. For each part, it is necessary to solve the homogeneous equation:

$$
D^{*}\left[\frac{\partial^{4}}{\partial x^{4}}-2\left(\frac{n}{R}\right)^{2} \frac{\partial^{2}}{\partial x^{2}}+\left(\frac{n}{R}\right)^{4}\right] \tilde{W}_{p}-\omega^{2} \rho_{e} h \tilde{W}_{p}=0, \quad p=1,2,
$$

where $\tilde{W}_{p}$ is the radial displacement of part $p$ of the shell for the circumferential order $n$. For each part $p$, the solutions of (35) take the form:

$$
\tilde{W}_{p}=A_{p} e^{r_{1} x}+B_{p} e^{r_{2} x}+C_{p} e^{r_{3}^{+1-} x}+D_{p} e^{r_{4}^{+1-} x}, \quad p=1,2
$$

where $A_{p}, B_{p}, C_{p}, D_{p}$ are constants to be defined and $r_{1}, r_{2}, r_{3}^{+/-}, r_{4}^{+/-}$are the roots of the characteristic equation of (35): 
$\cdot r_{1}=\sqrt{\left(\frac{n}{R}\right)^{2}+\left(\frac{\omega^{2} \rho_{e} h}{D^{*}}\right)^{2}}$

$\cdot r_{2}=-\sqrt{\left(\frac{n}{R}\right)^{2}+\left(\frac{\omega^{2} \rho_{e} h}{D^{*}}\right)^{2}}$

• if $\frac{n}{R} \geq \frac{\omega^{2} \rho_{e} h}{D^{*}}$, then

$r_{3}^{+}=\sqrt{\left(\frac{n}{R}\right)^{2}-\left(\frac{\omega^{2} \rho_{e} h}{D^{*}}\right)^{2}}$ and $r_{4}^{+}=-\sqrt{\left(\frac{n}{R}\right)^{2}-\left(\frac{\omega^{2} \rho_{e} h}{D^{*}}\right)^{2}}$

- if $\frac{n}{R}<\frac{\omega^{2} \rho_{e} h}{D^{*}}$, then

$$
r_{3}^{-}=j \sqrt{\left(\frac{\omega^{2} \rho_{e} h}{D^{*}}\right)^{2}-\left(\frac{n}{R}\right)^{2}} \text { and } r_{4}^{-}=-j \sqrt{\left(\frac{\omega^{2} \rho_{e} h}{D^{*}}\right)^{2}-\left(\frac{n}{R}\right)^{2}} .
$$

As the considered shell has an infinite length, there is no reflexion at the extremities of the shell, therefore:

$$
A_{1}=0, C_{1}=0, B_{2}=0, D_{2}=0 .
$$

At the junction between the two parts, we obain:

$$
\begin{aligned}
& \tilde{W}_{1}=\tilde{W}_{2} \\
& \frac{\partial \tilde{W}_{1}}{\partial x}=\frac{\partial \tilde{W}_{2}}{\partial x} \\
& \tilde{M}_{1}=\tilde{M}_{2}+\tilde{M} \\
& \tilde{T}_{1}=\tilde{T}_{2}+\tilde{F}
\end{aligned} \mid \text { at } x=0,
$$

where $\tilde{M}_{p}$ and $\tilde{T}_{p}$ are, respectively, the flexural moment (about the $\theta$-axis) and the shear force (about the $r$-axis) of the part $p$ of the shell for the circumferential order $n$. 
With the Love- Kirchhoff assumptions, the flexural moment and the shear force from the radial displacement can be expressed as:

$$
\begin{aligned}
& \tilde{M}_{p}=D^{*}\left[\frac{\partial^{2} \tilde{W}_{p}}{\partial x^{2}}-v\left(\frac{n}{R}\right)^{2} \tilde{W}_{p}\right], p=1,2, \text { and }, \\
& \tilde{T}_{p}=D^{*}\left[\frac{\partial^{3} \tilde{W}_{p}}{\partial x^{3}}-(2-v)\left(\frac{n}{R}\right)^{2} \frac{\partial \tilde{W}_{p}}{\partial x}\right], p=1,2 .
\end{aligned}
$$

From Eq. (36) and Eq. (41-44), we obtain the matrix equation:

$$
\left[\begin{array}{l}
A_{2} \\
B_{1} \\
C_{2} \\
D_{1}
\end{array}\right]=\left[\begin{array}{cccc}
1 & -1 & 1 & -1 \\
r_{1} & r_{1} & r_{3}^{+/-} & r_{3}^{+/-} \\
\varsigma_{1} & -\varsigma_{1} & \varsigma_{3}^{+/-} & -\varsigma_{3}^{+--} \\
\tau_{1} & -\tau_{2} & \tau_{3}^{+/-} & -\tau_{4}^{+/-}
\end{array}\right]^{-1}\left[\begin{array}{c}
0 \\
0 \\
\tilde{M} \\
\tilde{F}
\end{array}\right]
$$

where:

$$
\begin{gathered}
\varsigma_{1}=D^{*}\left[r_{1}^{2}-v\left(\frac{n}{R}\right)^{2}\right], \varsigma_{3}^{+/-}=D^{*}\left[r_{3}^{+/ L^{2}}-v\left(\frac{n}{R}\right)^{2}\right], \\
\tau_{1}=D^{*}\left[r_{1}^{3}-(2-v)\left(\frac{n}{R}\right)^{2} r_{1}\right], \tau_{2}=D^{*}\left[r_{2}^{3}-(2-v)\left(\frac{n}{R}\right)^{2} r_{2}\right], \\
\tau_{3}^{+/-}=D^{*}\left[r_{3}^{+/-^{3}}-(2-v)\left(\frac{n}{R}\right)^{2} r_{3}^{+/-}\right], \tau_{4}^{+/-}=D^{*}\left[r_{4}^{+/{ }^{3}}-(2-v)\left(\frac{n}{R}\right)^{2} r_{4}^{+/-}\right] .
\end{gathered}
$$

Analytical expressions of $A_{2}, B_{1}, C_{2}, D_{1}$ can be easily deduced from this system. Then, for each circumferential order $n$, it is possible to write the radial displacements of the shell: 


$$
\left\{\begin{array}{l}
\tilde{W}_{1}=B_{1} e^{r_{2} x}+D_{1} e^{r_{4}^{+/-} x}, x \leq 0, \\
\tilde{W}_{2}=A_{2} e^{r_{1} x}+C_{2} e^{r_{3}^{+/-} x}, x>0 .
\end{array}\right.
$$

and the angular rotation about $\theta$-axis:

$$
\left\{\begin{array}{l}
\tilde{\varphi}_{1}=B_{1} r_{2} e^{r_{2} x}+D_{1} r_{4}^{+/-} e^{r_{4}^{+l-} x}, x \leq 0, \\
\tilde{\varphi}_{2}=A_{2} r_{1} e^{r_{1} x}+C_{2} r_{3}^{+l-} e^{r_{3}^{++-} x}, x>0 .
\end{array}\right.
$$

Considering these results in the case $\tilde{F}=1$ and $\tilde{M}=0$, we can finally calculate $\tilde{Y}_{W_{i} F_{j}}^{\text {shell }}$ and $\tilde{Y}_{\varphi_{i} F_{j}}^{\text {shell }}$ using the relations:

$$
\begin{aligned}
& \forall(i, j) \in([1, \Theta])^{2} / x_{i}-x_{j} \leq 0 \Rightarrow \tilde{Y}_{W_{i} F_{j}}^{\text {shell }}=\tilde{W}_{1}\left(x_{i}-x_{j}, n\right), \tilde{Y}_{\varphi_{i} F_{j}}^{\text {shell }}=\tilde{\varphi}_{1}\left(x_{i}-x_{j}, n\right), \\
& \forall(i, j) \in([1, \Theta])^{2} / x_{i}-x_{j}>0 \Rightarrow \tilde{Y}_{W_{i} F_{j}}^{\text {shell }}=\tilde{W}_{2}\left(x_{i}-x_{j}, n\right), \tilde{Y}_{\varphi_{i} F_{j}}^{\text {shell }}=\tilde{\varphi}_{2}\left(x_{i}-x_{j}, n\right) .
\end{aligned}
$$

The same process may be used to calculate $\tilde{Y}_{W_{i} M_{j}}^{\text {shell }}$ and $\tilde{Y}_{\varphi_{i} M_{j}}^{\text {shell }}$ with the case $\tilde{F}=0$ and $\tilde{M}=1$.

\subsection{RESOLUTION WITH AN ACCELERATED SPECTRAL APPROACH}

In Sec. 4.2, we described a spectral approach using a sampling wavenumber $\bar{k}_{x}$ ensuring us that the high displacement amplitudes are taken into account in the inverse Fourier transform. However, the decrease of the spectral displacements about variable $k_{x}$ varies as a function of the displacement variable and the direction of the excitation. In particular, the decrease is slower for the spectral rotation for a moment excitation, $\widetilde{\widetilde{\varphi}}_{M}$ than the spectral radial displacement for a radial force excitation, $\tilde{\widetilde{W}}_{F}$. This fact can be illustrated if we neglect the effects of the curvature 
of the shell and the couplings between the axial/torsional waves and the flexural waves (i.e. Love-Kircchoff assumptions). In this case, we can write:

$$
\begin{aligned}
& \tilde{\tilde{W}}_{F}\left(k_{x}, n\right)=\frac{1}{D^{*}\left[k_{x}{ }^{2}+\left(\frac{n}{R}\right)^{2}\right]^{2}-\rho \omega^{2} h+Z_{\text {fluid }}}, \text { and, } \\
& \tilde{\tilde{\varphi}}_{M}\left(k_{x}, n\right)=-\frac{k_{x}^{2}}{D^{*}\left[k_{x}{ }^{2}+\left(\frac{n}{R}\right)^{2}\right]^{2}-\rho \omega^{2} h+Z_{\text {fluid }}}
\end{aligned}
$$

These expressions result in $\tilde{\widetilde{\varphi}}_{M}=O\left(\frac{1}{k_{x}^{2}}\right)$ and $\tilde{\widetilde{W}}_{F}=O\left(\frac{1}{k_{x}^{4}}\right)$. The decrease of $\tilde{\widetilde{W}}_{F}$ about $\frac{1}{k_{x}^{4}}$ is sufficiently high to obtain an accurate result by truncating the wavenumber space by the sampling wavenumber $\bar{k}_{x}$ whereas the decrease of $\widetilde{\widetilde{\varphi}}_{M}$ about $\frac{1}{k_{x}^{2}}$ is not always sufficient.

In order to improve convergence about $k_{x}$, we propose using the analytic result of the Sec. 4.3 to represent the components in the "high" wavenumber domain. To do this, we consider the quantity $\Omega_{M}$ defined by:

$$
\Omega_{M}(x, \theta)=\varphi_{M}(x, \theta)-\psi_{M}(x, \theta),
$$

where $\varphi_{M}$ and $\psi_{M}$ are both the angular rotations when the shell is excited by a moment. For the first variable, $\varphi_{M}$, the fluid loading is fully taken into account and the shell is modelled by the Flügge equations (i.e. the model of Sec. 4.2), whereas for the second variable, $\psi_{M}$, the fluid loading is approximated by its added mass effect and the shell is modelled by the Love-Kircchoff assumptions (i.e. the model of Sec. 4.3). 
Thus $\tilde{\varphi}_{M}$ is given by:

$$
\tilde{\varphi}_{M}=\tilde{\Omega}_{M}+\tilde{\psi}_{M}
$$

where $\tilde{\psi}_{M}$ is obtained by the analytic Fourier transform of $\psi_{M}$ (result of Sec. 4.3), and, $\widetilde{\Omega}_{M}$ may be obtained numerically by a discrete inverse Fourier transform of $\tilde{\widetilde{\Omega}}_{M}$ using the sampling wavenumber $\bar{k}_{x}$. The decrease of $\widetilde{\widetilde{\Omega}}_{M}$ about $k_{x}$ is greater than the decrease of $\tilde{\widetilde{\varphi}}_{M}$, as illustrated in Fig. 6. Therefore the difficulty observed when calculating $\tilde{\varphi}_{M}$ from $\tilde{\widetilde{\varphi}}_{M}$ does not occur when $\widetilde{\Omega}_{M}$ is calculated from $\widetilde{\widetilde{\Omega}}_{M}$ which is the advantage of the approach presented in this section. It can be seen that $\tilde{\Omega}_{M}$ can be considered as the error when estimating $\tilde{\varphi}_{M}$ from $\tilde{\psi}_{M}$ (i.e. from the simplified model of Sec. 4.3).

An illustration of the acceleration of convergence in the wavenumber space is shown in Fig. 7 where we plotted the admittances of a shell calculated with and without acceleration for different values of $\bar{k}_{x}$. Without the acceleration (i.e. method 1), the results converge slowly and the calculation requires $\bar{k}_{x}>200 \mathrm{~m}^{-1}$ to converge correctly, whereas the calculation with the acceleration (i.e. method 3) converges with $\bar{k}_{x}=25 \mathrm{~m}^{-1}$ only.

This technique can be applied to estimate $\tilde{Y}_{W F}, \tilde{Y}_{\theta F}$, and $\tilde{Y}_{W M}$ although the convergence in the wavenumber is not a matter for these quantities. 


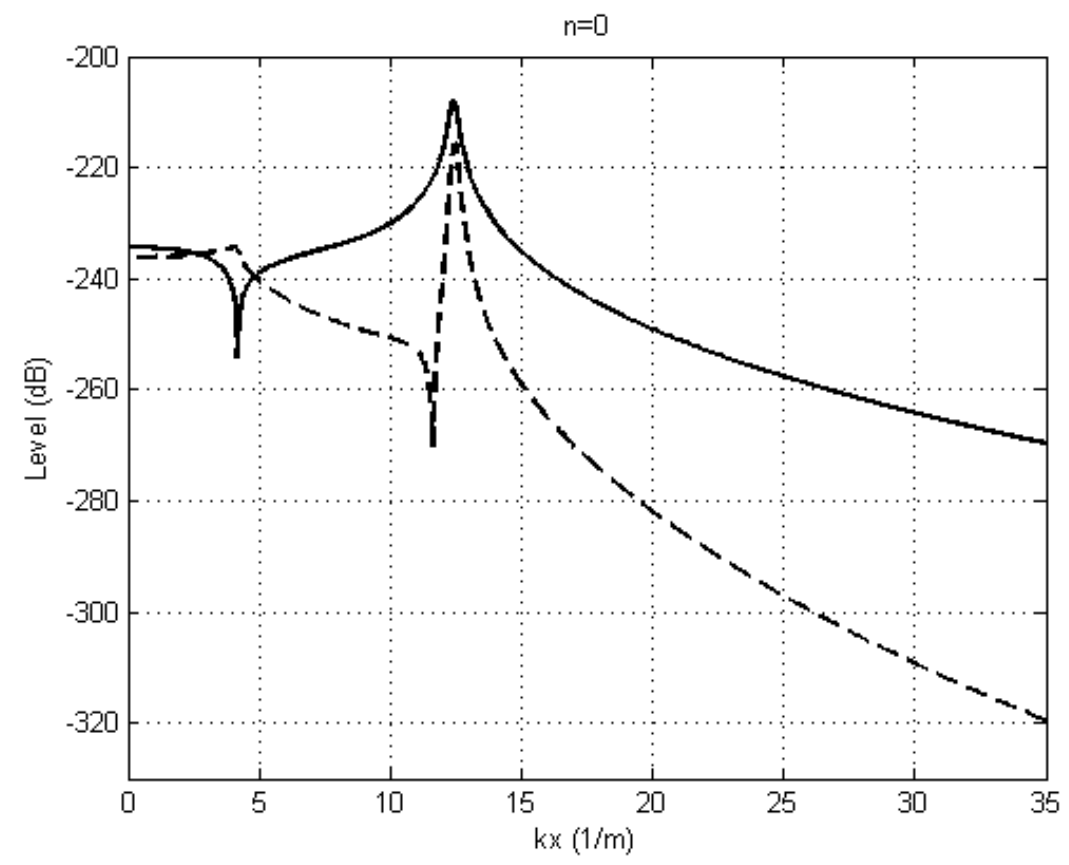

FIG. 6: Example of spectrum for $\widetilde{\widetilde{\varphi}}_{M}\left(k_{x}, 0\right)$ (solid line) and $\widetilde{\widetilde{\Omega}}_{M}\left(k_{x}, 0\right)$ (dashed line). Results at $1000 \mathrm{~Hz}\left(\mathrm{~dB}\right.$, ref. $\left.1 \mathrm{~m} \cdot \mathrm{N}^{-1}\right)$. Steel shell of $5 \mathrm{~m}$ radius and $30 \mathrm{~mm}$ thick immerged in water $(\eta=0.05)$ 


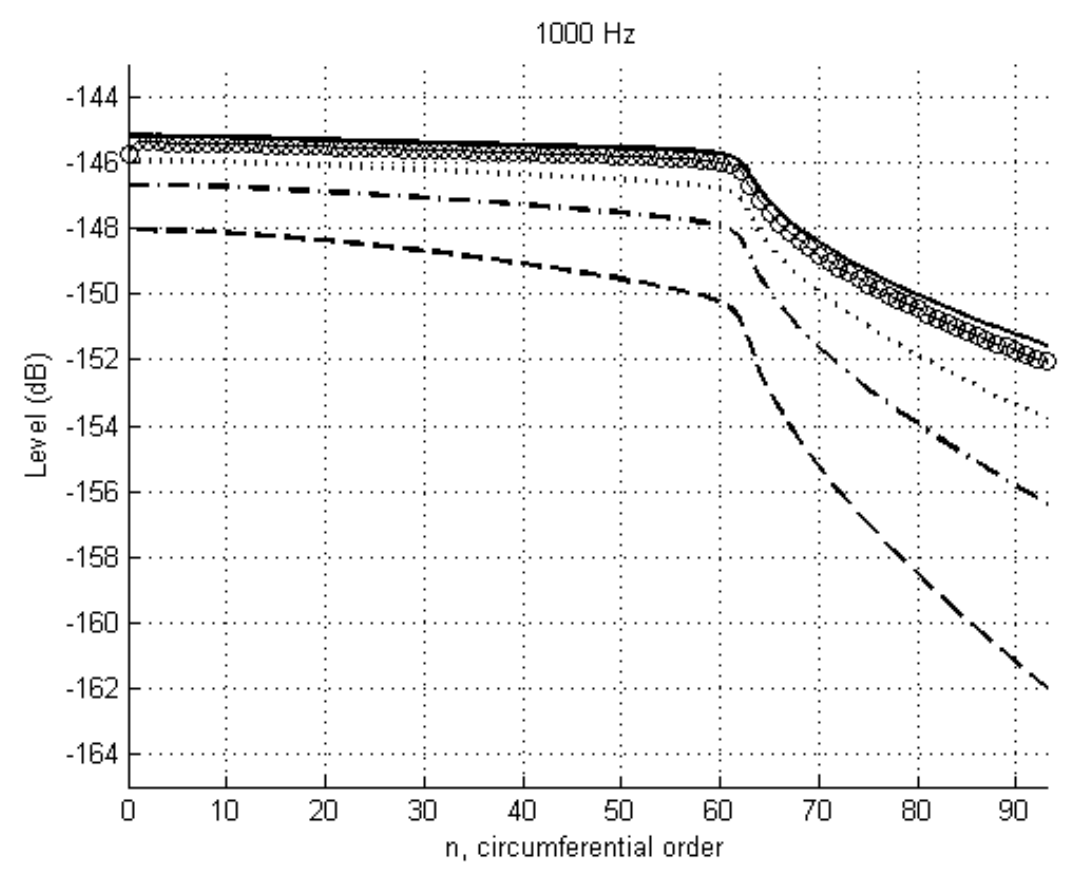

FIG. 7: Comparison of the amplitudes of the input admittance $\tilde{Y}_{\theta M}$ for different values of the sampling wavenumber $\bar{k}_{x}$ and with/without the acceleration of convergence: method 1 with $\bar{k}_{x}=25 \mathrm{~m}^{-1}$, dashed line; method 1 with $\bar{k}_{x}=50 \mathrm{~m}^{-1}$, dashed-dotted line; method 1 with $\bar{k}_{x}=100 \mathrm{~m}^{-1}$, dotted line; method 1 with $\bar{k}_{x}=200 \mathrm{~m}^{-1}$, circle markers; method 3 with $\bar{k}_{x}=25 \mathrm{~m}^{-1}$, solid line. Results at $1000 \mathrm{~Hz}\left(\mathrm{~dB}\right.$, ref. $\left.1 \mathrm{~N}^{-1}\right)$. Steel shell of $5 \mathrm{~m}$ radius and 30 mm thick immerged in water $(\eta=0.05)$.

\section{VIBRATION AND SOUND RADIATION FROM THE FLUID LOADED SHELL COUPLED WITH ITS INTERNAL FRAMES}

Approaches were presented in Sec. 3 and 4 to estimate the circumferential admittances and the free displacements for the internal frames and for the shell, respectively. These calculations should be performed for circumferential orders lower than a maximal circumferential order $\bar{N}$. The criterion for defining $\bar{N}$ can be based on the same principle used to define the sampling 
wavenumber criterion in Sec. 4.2. (see [34] for more details) Indeed, $\bar{N}$ may be seen as the sampling wavenumber about the circumference of the shell $\left(\bar{N}=\bar{k}_{\theta}\right)$ and may defined from the natural wavenumbers of the shell, the frames and the acoustic fluid. However, the natural wavenumbers of the internal frames cannot be calculated easily due to their complex geometries. Therefore we analyse the curves of the circumferential admittances of the internal frames numerically to determine a maximal circumferential order $\bar{n}_{i}$ associated with the $i^{\text {th }}$ internal frame. A numerical process allows us to determine the position of the peaks (i.e. resonances) of the admittances. These positions are defined on the circumferential order axis. Taking $\bar{n}_{i}$ as an upper bound of these positions ensures that the resonant behaviour of the frame will be taken into account correctly in the model.

Thus it is possible to define the maximal circumferential order $\bar{N}$ by:

$$
\bar{N}=\operatorname{int}\left[\kappa_{y} \max \left[R k_{l}, R k_{t}, R k_{f}, R k_{0}, \bar{n}_{i \in[1, \Theta]}\right]\right]+1
$$

where $\kappa_{y}$ is a margin coefficient (by default, therefore $\kappa_{y}=1.5$ ).

The forces/moments exerted by the frames on the shell can be calculated by using Eq. (5) for each circumferential order $n \in[0, \bar{N}]$. These forces are then injected in the model of the cylindrical shell of the Sec. 4.2. In this case, the spectral forces are given by:

$$
\left\{\begin{array}{l}
\tilde{\widetilde{F}}_{U U}=-\frac{\left(1-v^{2}\right) R^{2}}{E h} \sum_{i=1}^{\Theta} e^{-j k_{x} x_{i}} \tilde{L}_{i}^{\text {frame }}, \\
\tilde{\widetilde{F}}_{V V}=-\frac{\left(1-v^{2}\right) R^{2}}{E h} \sum_{i=1}^{\Theta} e^{-j k_{x} x_{i}} \tilde{T}_{i}^{\text {frame }}, \\
\tilde{\widetilde{F}}_{W W}=\frac{\left(1-v^{2}\right) R^{2}}{E h} \sum_{i=1}^{\Theta} e^{-j k_{x} x_{i}}\left(\tilde{F}_{i}^{\text {frame }}+j k_{x} \tilde{M}_{i}^{\text {frame }}\right) .
\end{array}\right.
$$


If the external load is directly applied on the cylindrical shell, the spectral forces due to this load should be added to the previous equation.

The spectral displacements of the shell coupled with its frames are calculated by using Eq. (17) and the spectral forces of Eq. (56). We deduce the displacement field in the physical space with a 2-D inverse discrete Fourier transform:

$$
\widetilde{\xi}\left(k_{x}, k_{\theta}\right) \stackrel{2 D \text { I.D.F.T. }}{\longrightarrow} \xi(x, \theta)
$$

The radiated pressure in the near field can be estimated with the same approach with Eq. (15) whereas the radiated pressure in the far field can be estimated with the stationary phase theorem [33].

\section{INFLUENCE OF THE SHELL ADMITTANCE CALCULATION ON THE RESULTS OF THE CIRCUMFERENTIAL ADMITTANCE APPROACH}

We propose to compare the results of the present approach when the shell admittances are obtained with the three different methods described in Sec. 4. To compare the results with another model, we consider only flexural motions of the shell (Love-Kircchoff assumptions) and the stiffeners are uniformly spaced. The reference result is then obtained by adapting for the shell the wavenumber approach described in [34] for the plate. We emphasize that the couplings of admittances are not used in this approach; the calculations are based on the use of the Poisson formula and the periodicity properties of the model.

For this comparison, the $5 \mathrm{~m}$ radius and $30 \mathrm{~mm}$ thick shell is assumed to be made of steel $\left(\rho=7800 \mathrm{~kg} / \mathrm{m}^{3} ; \mathrm{E}=2.1 .10^{11} \mathrm{~Pa} ; v=0.3, \eta=0.005\right)$ and immerged in water. The ring stiffeners 
are regularly spaced at $1.5 \mathrm{~m}$ along the axis of the shell and have a T-cross section $300 \mathrm{~mm} \times 60$ $\mathrm{mm} / 60 \mathrm{~mm}$ x $300 \mathrm{~mm}$. For CAA calculations, 80 stiffeners are considered whereas the reference model takes into account an infinite number of stiffeners. A harmonic point force is located at $x=0.4 \mathrm{~m}$ (i.e. between two stiffeners) and $\theta=0^{\circ}$.

Fig. 8 shows the displacement levels on the generating line of the cylinder $\theta=180^{\circ}$ (i.e. opposite side of the excitation). The CAA result obtained with the shell admittances of Sec. 4.2 (dotted line) is a poor approximation of the reference result (solid line). Only the displacement levels between the two stiffeners near the excitation position are approximately predicted by this calculation. This can be explained by the fact that the calculations of certain admittances $\left(\tilde{Y}_{\theta M}^{\text {shell }}\right.$ in particular) are not accurate enough. The shell admittances calculated analytically in Sec. 4.3 (dash-dotted line) give a better result but only the shell admittances obtained in Sec. 4.4 make it possible for CAA to give a very good result (dash line). Fig. 9 shows the values of $\tilde{Y}_{W F}^{\text {shell }}$ and $\tilde{Y}_{\theta M}^{\text {shell }}$ obtained with the three different methods. The differences observed in this figure explain the differences of the CAA results in Fig. 8. Indeed, it can be seen that the first method gives the same values as the third method for $\tilde{Y}_{W F}^{\text {shell }}$ but underestimates the values of $\tilde{Y}_{\theta M}^{\text {shell }}$ (as in Fig. 7). The rotational stiffness of the shell is therefore overestimated. Consequently, the reaction moments exerted by the frames on the shell are overestimated by CAA. On the other hand, the second method gives the same values as the third method for $\tilde{Y}_{\theta M}^{\text {shell }}$ but overestimates the values of $\tilde{Y}_{W F}^{\text {shell }}$ for the non resonant circumferential order. Although the calculation frequency is below the critical frequency, the fluid added mass assumption of the method 2 does not permit estimating the shell admittances with enough accuracy for CAA convergence. 

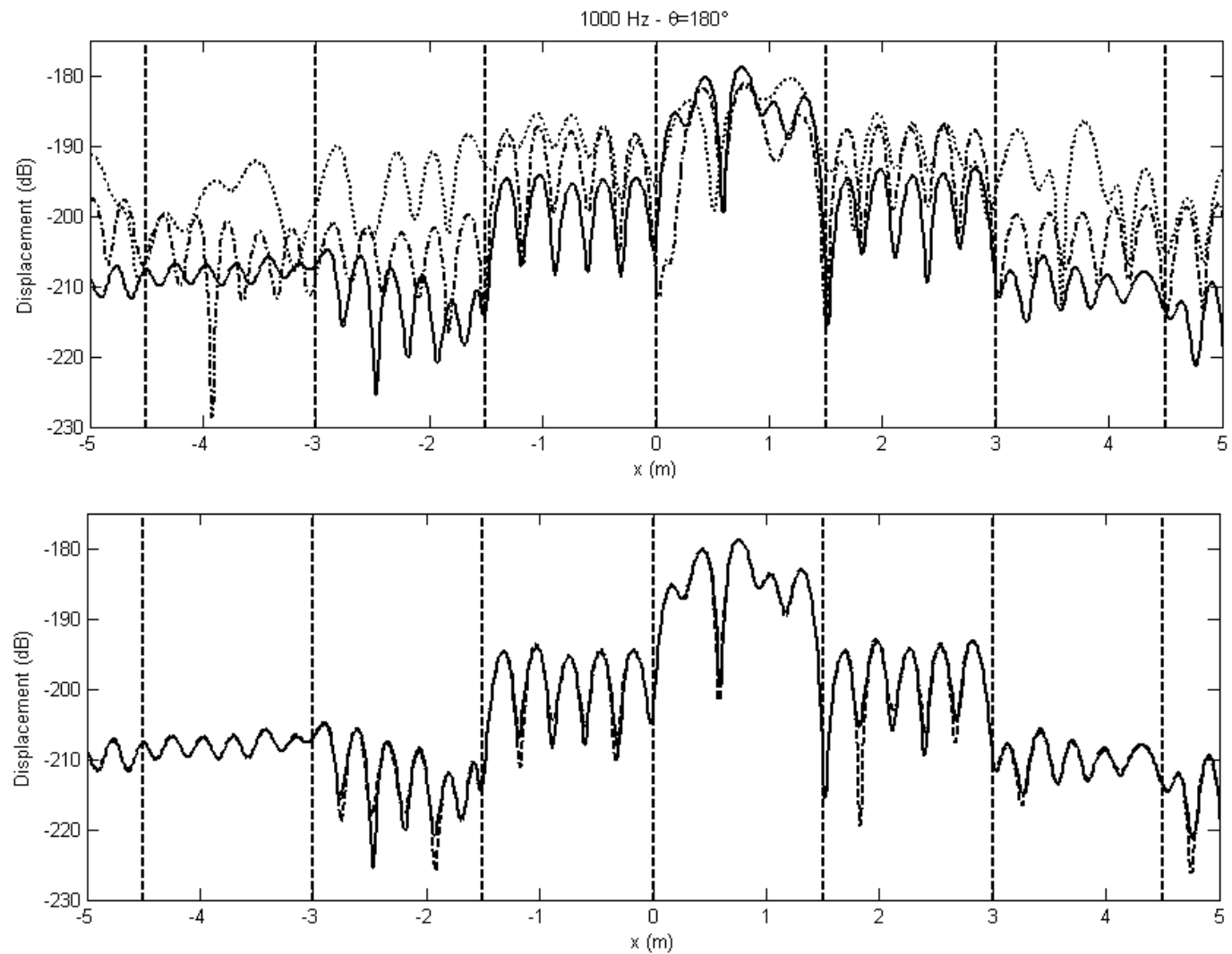

FIG. 8: Shell displacement level on the axis $\theta=180^{\circ}$. Results at $1000 \mathrm{~Hz}$ (stiffeners' position: vertical dash lines, $\mathrm{dB}$ ref. $1 \mathrm{~m} \cdot \mathrm{N}^{-1}$ ). Comparison of different calculations.

Upper part: Solid line, reference; dotted line, CAA with $\tilde{Y}^{\text {shell }}$ of Sec. 4.2; dash-dotted line, CAA with $\tilde{Y}^{\text {shell }}$ of Sec. 4.3; Lower part: Solid line, reference; dash line, CAA with $\tilde{Y}^{\text {shell }}$ of Sec. 4.4. 

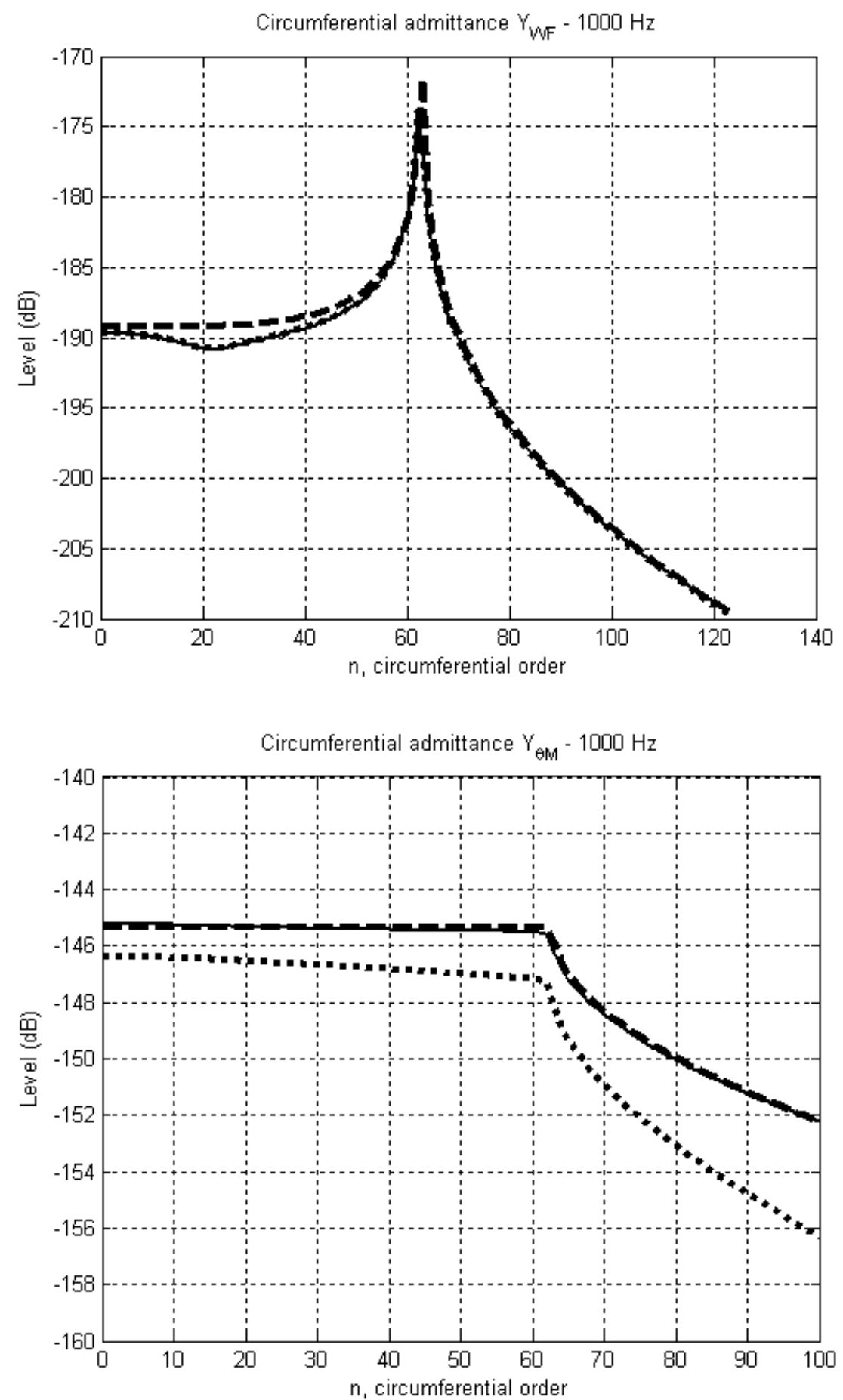

FIG. 9. Comparison of $\tilde{Y}_{W F}$ (upper, $\mathrm{dB}$ ref. $1 \mathrm{~m}^{2} . \mathrm{N}^{-1}$ ) and $\tilde{Y}_{\theta M}$ (lower, $\mathrm{dB}$ ref. $1 \mathrm{~N}^{-1}$ ) obtained by the three methods: solid line, Sec. 4.4; dashed line, Sec. 4.2; dashed-dotted line, Sec. 4.3. 


\section{EXAMPLE OF SUBMARINE APPLICATION}

In this section, we illustrate the present approach for a submarine test case. The case considered is shown in Fig. 10 and is composed of a $5 \mathrm{~m}$ radius $42.3 \mathrm{~m}$ length and a $30 \mathrm{~mm}$ thick cylindrical shell stiffened with 65 stiffeners and 2 spherical bulkheads ( $10 \mathrm{~mm}$ thick, $30 \mathrm{~m}$ curvature radius). The stiffeners have different sections (three types of stiffeners) and their spacing varies from 0.5 $\mathrm{m}$ to $0.75 \mathrm{~m}$ (see Fig. 10). The shell is made of steel $(\eta=0.005)$, immerged in water and excited by a radial point force on the web of the $24^{\text {th }}$ frame. Clamped boundary conditions are assumed at the ends of the shell ( $2 \mathrm{~m}$ before the first stiffener and the $2 \mathrm{~m}$ after the last stiffener). They are taken into account in the CAA calculation by introducing fictive frames with null circumferential admittances.

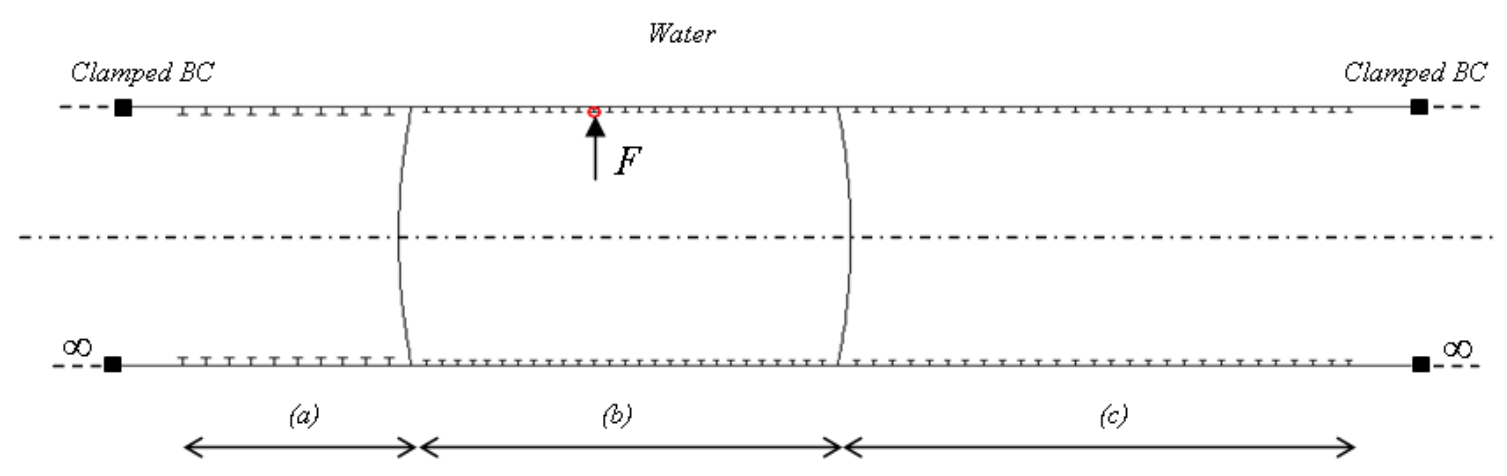

FIG. 10. Submarine hull application: Cylindrical shell: $5 \mathrm{~m}$ radius, $42.3 \mathrm{~m}$ length, $30 \mathrm{~mm}$ thick.

Stiffeners : (a), spacing $0.75 \mathrm{~m}$, T-cross-section (mm): 300x60/60x300; (b), spacing $0.5 \mathrm{~m}$, Tcross-section (mm): 200x15/15x200; (c), spacing: 0.6 m, T-cross-section (mm): 200x25/15x200.

An example of the radial displacements in the wavenumber space $\tilde{\tilde{W}}$ is given in Fig. 11. It is obtained directly from the coupling forces $\tilde{\mathbf{F}}^{\text {frame }}$ and the expression (17) of the spectral 
displacement, as described in Sec. 5. This kind of result facilitates understanding the behaviour of the shell, as proposed in reference [36], on the basis of the measurements. For example, it permits analysing the displacements that radiate in the far field: these displacements are contained in the supersonic region. It can also be used to predict the dominant directions of wave propagation. These directions [36] are given by identifying the regions of large displacement amplitudes having large curvature radii and drawing the normal to the curve in these regions. At $500 \mathrm{~Hz}$, we can predict multiple directions of propagation whereas at $2000 \mathrm{~Hz}$, two dominant directions exist.

The inverse space Fourier transform of the spectral displacements gives the displacements in physical space. Examples of results are given in Fig 12 in which the bulkhead positions are plotted in black. The same process can be used to estimate the radiated pressure in the near field whereas the stationary phase theorem permits deducing the radiated pressure in the far field.

In Fig. 12, it can be seen that at $500 \mathrm{~Hz}$ the vibratory field is almost homogenous in the excited compartment (i.e. part of the shell between two bulkheads). This is the result of the multiple directions of wave propagations as predicted previously from Fig. 11. The bulkheads lead to the attenuation of strong vibrations due to their considerable stiffness. At $2000 \mathrm{~Hz}$, the two directions of the dominant waves can be seen once again. They are due to the interaction between the cylindrical shell and the stiffeners that rigidify the shell along the circumference.

These results will be analyzed in greater detail in a future paper. They are presented here to illustrate the numerical approach. The computing times are reasonable (around 100 seconds at $500 \mathrm{~Hz}$ and around 250 seconds at $2000 \mathrm{~Hz}$ on a PC Pentium IV 3GHz monoprocessor). They increase with frequency as the maximal circumferential order $\bar{N}$ and the sampling wavenumber 
$\bar{k}_{x}$ increase with frequency. However the approach presented is able to predict the behaviour of a submarine hull up to several KHz. This has been made possible by assembling a dedicated model of the cylindrical shell immerged in water with the finite element models of its internal frames
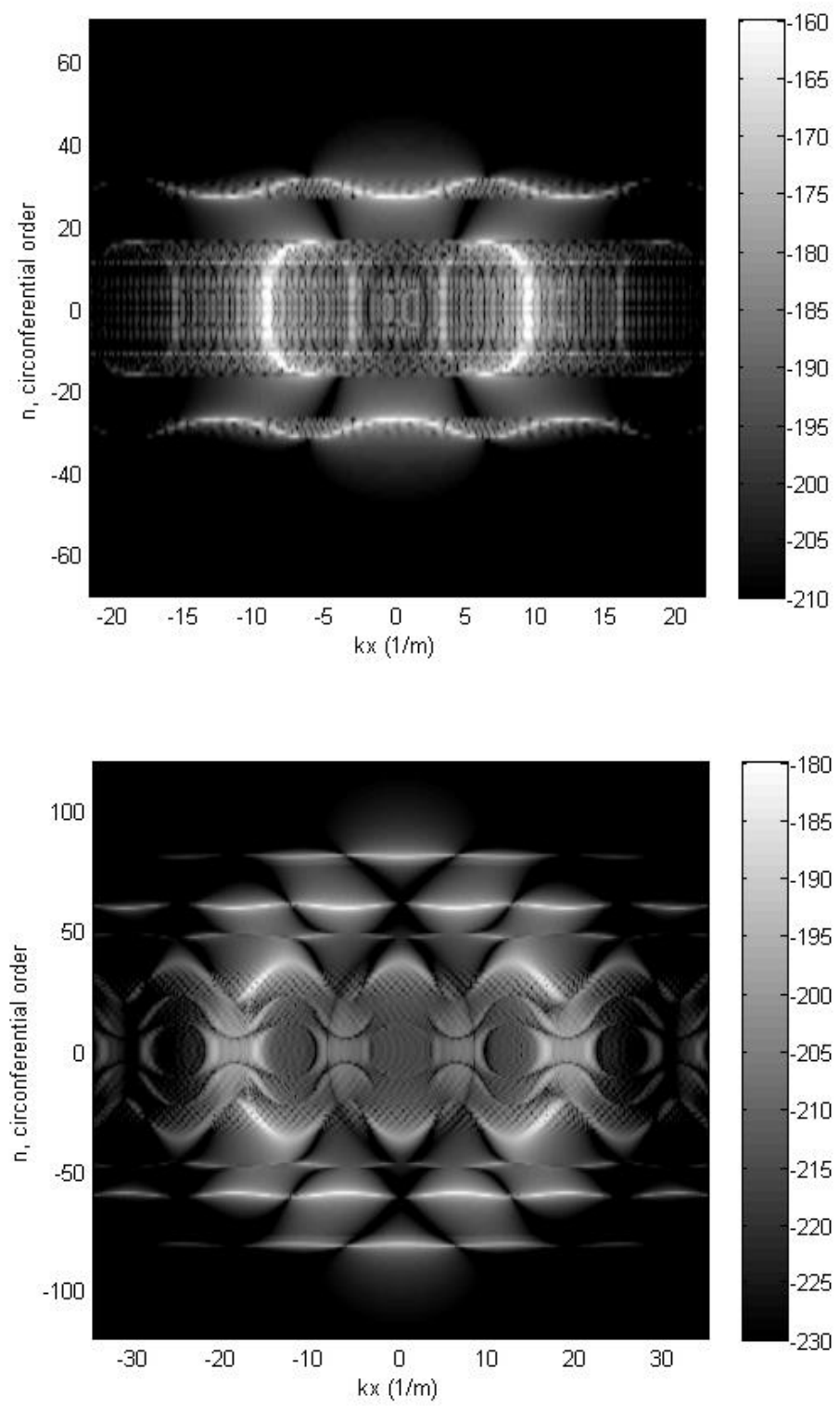

FIG. 11. Radial displacements of the shell $\left(\mathrm{dB}, \mathrm{ref} .1 \mathrm{~m}^{2} / \mathrm{N}\right)$ in the wavenumber space: upper, $500 \mathrm{~Hz}$; lower, $2000 \mathrm{~Hz}$. 

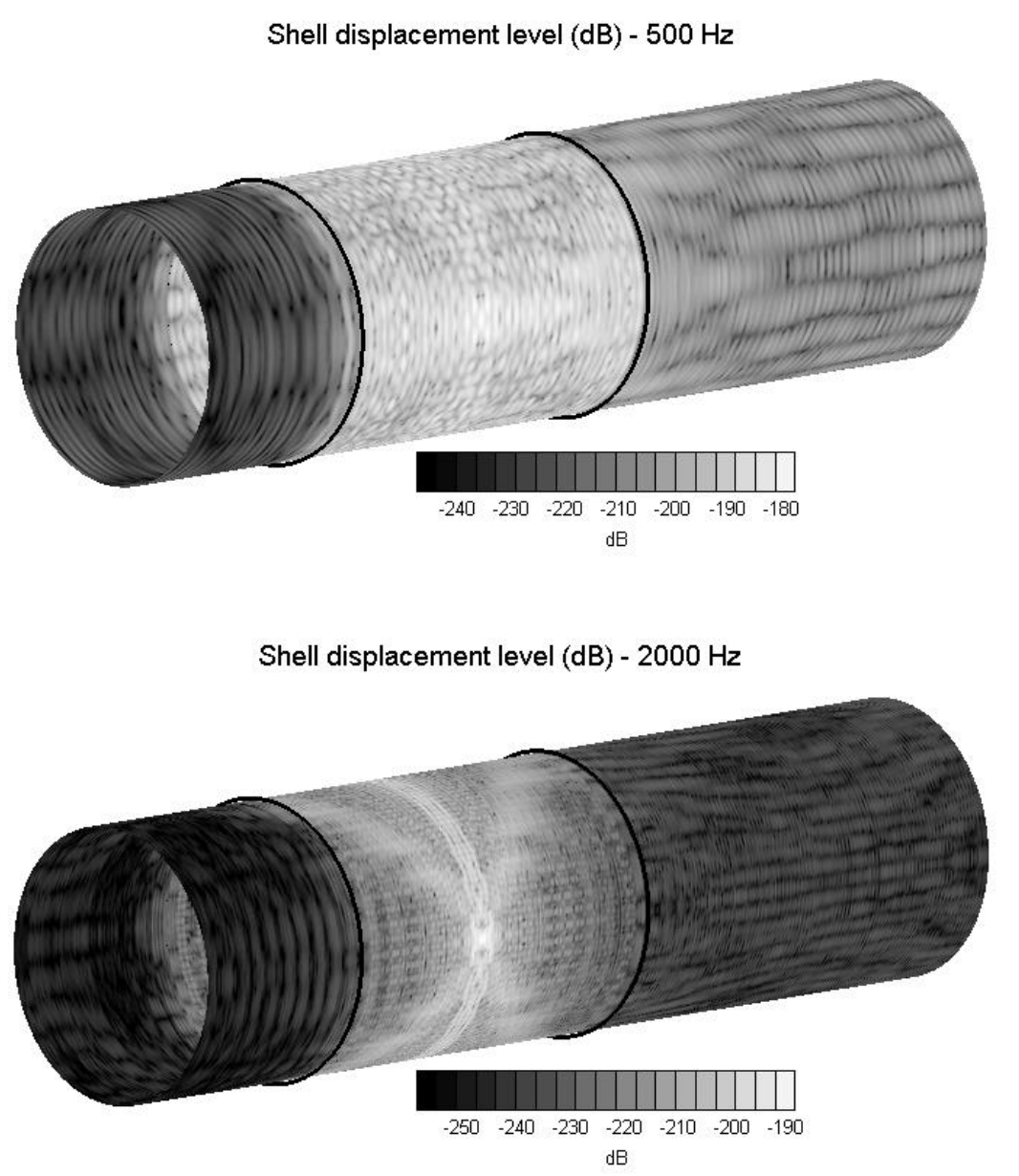

FIG. 12. Radial displacements levels of the shell (dB, ref. $\left.1 \mathrm{~m} . \mathrm{N}^{-1}\right)$ :

upper, $500 \mathrm{~Hz}$; lower, $2000 \mathrm{~Hz}$.

\section{CONCLUSIONS}

The circumferential admittance approach (CAA) presented in this paper is a powerful tool for assembling a numerical model of a fluid loaded shell with the finite element models of its internal frames. This approach consists in partitioning the problem so that the fluid loaded shell constitutes one subsystem and the frames constitute other subsystems. These subsystems are 
coupled together by assembling the circumferential admittances that characterised each subsystem independently. The Finite Element method can be used to evaluate the admittances of each type of internal frame. Three different approaches have been presented in this paper to estimate the shell admittances. It can be seen that the quality of the results of CAA strongly depends on the accuracy of the calculation of the shell admittances. The best results are obtained by an approach that combines the analytical result of a simplified shell problem and the numerical result that quantifies the difference between the simplified problem and the full problem. One has shown that the comparison of CAA results with a reference result on a fluid loaded uniformly stiffened shell is then quite good.

The intermediate results of this approach are the displacements and the pressures expressed in the wavenumber space. They are useful for understanding the physical characteristics involved in the acoustic radiation of the shell. This approach permits evaluating the vibratory field of a submarine pressure hull and its radiated pressure field, both in the near and far fields, within reasonable computing times. It will permit improving knowledge regarding acoustic stealth, target strength and self-noise problems in the submarines. It will be used to study the influence of the bulkheads, the ring-stiffeners, their spacing on the vibrations of the shell and its radiated pressure.

In the future, to validate definitely this approach, one should compare their results with reference ones which could be obtained in the low frequencies with finite element and boundary element methods ([5]). Comparisons with finite element calculations (NASTRAN code) had already been performed by the present authors on in vacuo stiffened shells (see some comparisons in [37]). Test cases were composed of shells with non uniformly spaced stiffeners and with axisymmetric bulkheads. Comparisons with CAA for radial or longitudinal excitations show good agreement between the two methods, as well as for radial, tangential and longitudinal displacements. 
Comparisons on cases of shells immersed in water (i.e. fluid loaded shell) are however necessary to validate definitely this approach.

\section{ACKNOWLEDGMENTS}

This work was carried out at the French Defence Agency (DGA/CTSN Toulon). The authors would like to thank this institution for authorizing them to publish these results. 


\section{REFERENCES}

[1] R.J. Urick, Principles of underwater sound. 3nd ed. (McGraw-Hill Book Company, New york, 1983).

[2] Q. Zhou and P.F. Joseph, "A numerical method for the calculation of dynamic response and acoustic radiation from an underwater structure," J. Sound Vib. 283, 853-873, (2005).

[3] Z. Tong, Y. Zhang, Z. Zhang and H. Hua, "Dynamic behavior and sound transmission analysis of a fluid-structure coupled system using the direct BEM/FEM,” J. Sound Vib. 299, 645-655 (2007).

[4] S. Merz, R. Kinns, N. Kessissoglou, "Structural and acoustic responses of a submarine hull due to propeller forces,” J. Sound Vib.325, 266-286 (2009).

[5] S. Merz, S. Oberst, P. G. Dylejko, N. J. Kessissoglou, Y. K. Tso and S. Marburg, "Development of coupled $\mathrm{FE} / \mathrm{BE}$ models to investigate the structural and acoustic responses of a submerged vessel," J. Comput. Acous. 15, 23-47 (2007).

[6] M.L. Rumerman, "Vibration and wave propagation in ribbed plates," J. Acoust. Soc. Am. 57, 370-373 (1975).

[7] B.R. Mace, "Periodically stiffened fluid-loaded plates, I: response to convected harmonic pressure and free wave propagation,” J. Sound Vib. 73(4), 473-486 (1980).

[8] G. Maidanik, "Response of ribbed panels to reverberant acoustic fields," J. Acoust. Soc. Am.Vol. 34, 809-826 (1962).

[9] R.S. Langley, "A dynamic stiffness technique for the vibration analysis of stiffened shell structures,” J. Sound Vib. 156(3), 521-540 (1992). 
[10] W. H. Hoppmann, II, "Some characteristics of the flexural vibrations of orthogonally stiffened cylindrical shells,” J. Acoust. Soc. Am.Vol, 30, 77-82 (1958).

[11] B. Laulagnet and J.L. Guyader, "Sound radiation by finite cylindrical ring-stiffened shell,” J. Sound Vib. 138, 173-191 (1990).

[12] J. Yan, T.Y. Li and T.G. Liu and J.X. Liu, "Characteristics of the vibrational power flow propagation in a submerged periodic ring-stiffened cylindrical shell," Appl. Acoust., 67, 550-569 (2006).

[13] J. Yan, T.Y. Li, J.X. Liu and X. Zhu, "Space harmonic analysis of sound radiation from a submerged periodic ring-stiffened cylindrical shell," Appl. Acoust., 67, p. 743755 (2006).

[14] C.H. Hodges, J. Power, and J. Woodhouse, "The low frequency vibration of a ribbed cylinder, part 1: theory; part 2: observations and interpretation,”. J. Sound Vib. 101, 219-256 (1985).

[15] W. Zhang, N. Vlahopoulos and $\mathrm{K}$. Wu, "An energy finite element formulation for high-frequency vibration analysis of externally fluid-loaded cylindrical shells with periodic circumferential sitffeners subjected to axi-symmetric excitation,” J. Sound Vib. 282, 679-700 (2005).

[16] D.M. Photiadis, J.A. Bucaro and B.H. Houston, "Scattering from flexural waves on a ribbed cylindrical shell," J. Acoust. Soc. Am. 96, 2785-2790 (1994).

[17] D.M. Photiadis, "Localization of helical flexural waves by irregularity," J. Acoust. Soc. Am. 96, 2291-2301 (1994).

[18] B.H. Houston, J.A. Bucaro and D.M. Photiadis, "Broadband acoustic scattering from a ribbed shell,” J. Acoust. Soc. Am.Vol. 98, 2851-2853 (1995). 
[19] M.H. Marcus, B.H. Houston and D.M. Photiadis, "Wave localization on a submerged cylindrical shell with rib aperiodicity," J. Acoust. Soc. Am. 109, 865-869 (2001).

[20] D.M. Photiadis and B.H. Houston, "Anderson localization of vibration on a framed cylindrical shell,” J. Acoust. Soc. Am.Vol. 106, 1377-1391 (1999).

[21] D.M. Photiadis, B.H. Houston, E.G. Williams and J.A. Bucaro, "Resonant response of complex shell structures,” J. Acoust. Soc. Am. 108, 1027-1035 (2000).

[22] X.W. Yin, L.J. Liu, R.X. Hua, R.Y. Shen, "Acoustic radiation from an infinite laminated composite cylindrical shell with doubly periodic rings," J. of Vib. and Acous. 131, 1-9 (2009).

[23] M. Tran-Van-Nhieu, "Scattering from a ribbed finite cylindrical shell," J. Acoust. Soc. Am. 110, 2858-2866 (2001).

[24] R.N. Miles, "An approximation method for modal analysis of finite periodic structures,”J. Sound Vib. 134, 165-174 (1989)

[25] R. Liétard, D. Décultot, G. Maze and M. Tran-Van-Nhieu, "Acoustic scattering from a finite cylindrical shell with evenly spaced stiffeners: Experimental investigation," J. Acoust. Soc. Am. 118, 2142-2146 (2005).

[26] M. Caresta and N.J. Kessissoglou, "Structural and acoustic responses of a fluid loaded cylindrical hill with structural discontinuities,” Appl. Acoust. 70, 954-963 (2009).

[27] M. Caresta, "Structural and acoustic responses of a submerged vessel,", PhD Thesis (University of New South Wales, 2009)

[28] X. Pan, Y. Tso and R. Juniper, "Active control of low-frequency hull-radiated noise," J. Sound Vib. 313, 29-45 (2008). 
[29] M. Zampolli, A. Tesei, F.B. Jensen, N. Malm, and J.B. Blottman III, “A computationally efficient finite element model with perfectly matched layers applied to scattering from axially symmetric objects," J. Acoust. Soc. Am. 122, 1472-1485 (2007).

[30] MSC NASTRAN 2001 - Reference Manual. (MSC.Software Corporation, Santa Ana, 2001)

[31] A.W. Leissa, Vibration of Shells. NASA SP-288 (1973).

[32] D.G. Karczub, "Expressions for direct evaluation of wave number in cylindrical shell vibration studies using the Flügge equations of motion,” J. Acoust. Soc. Am. 119, 3553- 3557 (2006).

[33] M.C. Junger and D. Feit, Sound, Structures and Their Interaction, 2nd ed. (MIT, Cambrige, MA, 1986).

[34] L. Maxit, "Wavenumber space and physical space responses of a periodically ribbed plate: A discrete approach,” Appl. Acoust. 70, 563-578 (2009).

[35] F. Fahy, Sound and Structural Vibration, $1^{\text {st }}$ ed. (Academic press, New York, 1985).

[36] D.M. Photiadis, E.G. Williams and B.H. Houston, "Wave-number space response of a near periodically ribbed shell,” J. Acoust. Soc. Am. 101, 877-886 (1996).

[37] L. Maxit, "Circumferential admittance approach for vibro-acoustic prediction of a submerged cylindrical shell with rib aperiodicity and transversal bulkheads," Proceedings of Acoustics'08, Paris, France, 2008. 


\section{Figure captions}

FIG. 1. (a), Illustration of the framed shell immerged in water ; (b), Partitioning and coupling forces.

FIG. 2. Deformation of a stiffener cross-section for a moment excitation. $1000 \mathrm{~Hz}, \mathrm{n}=0$.

T section: $300 \mathrm{~mm}$ x $60 \mathrm{~mm} / 60 \mathrm{~mm}$ x $300 \mathrm{~mm} . \mathrm{R}=5 \mathrm{~m}$. Material: $\mathrm{steel}(\eta=0.005)$.

FIG. 3. Comparison of the circumferential admittances $\tilde{Y}_{\theta M}^{\text {frame }}$ calculated with NASTRAN (full line) and with the 1D-beam model (dash line). $1000 \mathrm{~Hz}\left(\mathrm{~dB}\right.$ ref. $\left.1 \mathrm{~N}^{-1}\right)$.

FIG. 4. Infinite cylindrical shell immersed in water and excited by line excitations at $x=0$.

FIG. 5. Example of the radial displacement level $\left(\mathrm{dB}\right.$, ref. $\left.1 \mathrm{~m}^{2} . \mathrm{N}^{-1}\right)$ in the wavenumber domain. Steel shell of $5 \mathrm{~m}$ radius and $30 \mathrm{~mm}$ thick immerged in water $(\eta=0.005)$. Case of an axial load.

FIG. 6: Example of spectrum for $\widetilde{\widetilde{\varphi}}_{M}\left(k_{x}, 0\right)$ (solid line) and $\widetilde{\widetilde{\Omega}}_{M}\left(k_{x}, 0\right)$ (dashed line). Results at $1000 \mathrm{~Hz}\left(\mathrm{~dB}\right.$, ref. $\left.1 \mathrm{~m} \cdot \mathrm{N}^{-1}\right)$. Steel shell of $5 \mathrm{~m}$ radius and $30 \mathrm{~mm}$ thick immerged in water $(\eta=0.05)$. 
FIG. 7: Comparison of the amplitudes of the input admittance $\tilde{Y}_{\theta M}$ for different values of the sampling wavenumber $\bar{k}_{x}$ and with/without the acceleration of convergence: method 1 with $\bar{k}_{x}=25 \mathrm{~m}^{-1}$, dashed line; method 1 with $\bar{k}_{x}=50 \mathrm{~m}^{-1}$, dashed-dotted line; method 1 with $\bar{k}_{x}=100 \mathrm{~m}^{-1}$, dotted line; method 1 with $\bar{k}_{x}=200 \mathrm{~m}^{-1}$, circle markers; method 3 with $\bar{k}_{x}=25 \mathrm{~m}^{-1}$, solid line. Results at $1000 \mathrm{~Hz}\left(\mathrm{~dB}\right.$, ref. $\left.1 \mathrm{~N}^{-1}\right)$. Steel shell of $5 \mathrm{~m}$ radius and 30 mm thick immerged in water $(\eta=0.05)$.

FIG. 8: Shell displacement level on the axis $\theta=180^{\circ}$. Results at $1000 \mathrm{~Hz}$ (stiffeners' position: vertical dash lines, dB ref. $\left.1 \mathrm{~m} \cdot \mathrm{N}^{-1}\right)$. Comparison of different calculations. Upper part: Solid line, reference; dotted line, CAA with $\tilde{Y}^{\text {shell }}$ of Sec. 4.2; dash-dotted line, CAA with $\tilde{Y}^{\text {shell }}$ of Sec. 4.3; Lower part: Solid line, reference; dash line, CAA with $\tilde{Y}^{\text {shell }}$ of Sec. 4.4.

FIG. 9. Comparison of $\tilde{Y}_{W F}$ (upper, dB ref. $1 \mathrm{~m}^{2} . \mathrm{N}^{-1}$ ) and $\tilde{Y}_{\theta M}$ (lower, dB ref. $1 \mathrm{~N}^{-1}$ ) obtained by the three methods: solid line, Sec. 4.4; dashed line, Sec. 4.2; dashed-dotted line, Sec. 4.3.

FIG. 10. Submarine hull application: Cylindrical shell: $5 \mathrm{~m}$ radius, $42.3 \mathrm{~m}$ length, $30 \mathrm{~mm}$ thick. Stiffeners : (a), spacing $0.75 \mathrm{~m}, \mathrm{~T}$-cross-section (mm): 300x60/60x300; (b), spacing $0.5 \mathrm{~m}, \mathrm{~T}$ cross-section (mm): 200x15/15x200; (c), spacing: 0.6 m, T-cross section (mm): 200x25/15x200.

FIG. 11. Radial displacements of the shell $\left(\mathrm{dB}\right.$, ref. $\left.1 \mathrm{~m}^{2} / \mathrm{N}\right)$ in the wavenumber space: upper, $500 \mathrm{~Hz}$; lower, $2000 \mathrm{~Hz}$. 
FIG. 12. Radial displacements levels of the shell (dB, ref. 1 m. $\left.\mathrm{N}^{-1}\right)$ :

upper, $500 \mathrm{~Hz}$; lower, $2000 \mathrm{~Hz}$. 